\title{
REMARKS ON FUJIWARA'S STATIONARY PHASE METHOD ON A SPACE OF LARGE DIMENSION WITH A PHASE FUNCTION INVOLVING ELECTROMAGNETIC FIELDS
}

\section{TETSUO TSUCHIDA}

\section{Introduction}

We consider an oscillatory integral of the form

$$
\begin{aligned}
& I\left(\left\{t_{j}\right\}, S, a, \nu\right)\left(x_{L}, x_{0}\right)= \\
& \quad \prod_{j=1}^{L}\left(\frac{\nu i}{2 \pi t_{j}}\right)^{d / 2} \int_{\mathbf{R}^{d(L-1)}} e^{-i \nu S\left(x_{L}, \cdots, x_{0}\right)} a\left(x_{L}, \cdots, x_{0}\right) \prod_{j=1}^{L-1} d x_{j} .
\end{aligned}
$$

Here each $x_{j}, j=0,1, \ldots, L$, runs in $\mathbf{R}^{d}, \nu>1$ is a constant and $t_{j}, j=1, \ldots$, $L$, are positive constants. Fujiwara [5] discussed this integral for $L$ large and developed the stationary phase method with an estimate of the remainder term for the phase function $S\left(x_{L}, \ldots, x_{0}\right)$ coming from the action integral for a particle in an electric field. But his results cannot be applied to the integral which naturally arises in the discussion of quantum mechanics of a charged particle moving in a magnetic field. In this paper we extend his results to the case for the phase function involving both electric and magnetic fields.

We denote the $l$-th component of $x \in \mathbf{R}^{d}$ by $(x)_{l}$, and use the notations: $\partial_{j}^{\alpha}=$ $\partial_{x j}^{\alpha}=\partial_{\left(x_{j}\right)_{1}}^{\alpha_{1}} \cdots \partial_{\left(x_{j}\right)_{d}}^{\alpha_{d}}$ with a multi-index $\alpha=\left(\alpha_{1}, \ldots, \alpha_{d}\right)$, and $\partial_{j} f\left(x_{j}\right)=\partial_{x_{j}} f\left(x_{j}\right)$ as the gradient of $f\left(x_{j}\right)$.

Our assumption for the phase function $S\left(x_{L}, \ldots, x_{0}\right)$ is the following: (H.1) $S\left(x_{L}, \ldots, x_{0}\right)$ is a real-valued function of the form

$$
S\left(x_{L}, \ldots, x_{0}\right)=\sum_{j=1}^{L} S_{j}\left(t_{j}, x_{j}, x_{j-1}\right),
$$

where

Received September 28, 1993. 


$$
S_{j}\left(t_{j}, x_{j}, x_{j-1}\right)=\frac{\left|x_{j}-x_{j-1}\right|^{2}}{2 t_{j}}+\omega_{j}\left(t_{j}, x_{j}, x_{j-1}\right), j=1, \ldots, L,
$$

and $\omega_{j}\left(t_{j}, x_{j}, x_{j-1}\right)$ satisfies the following conditions:

(i) For any $m \geq 2$ there exists a constant $\kappa_{m}>0$ independent of $j$ and $t_{j}$ such that

$$
\max _{2 \leq|\alpha+\beta| \leq m} \sup _{x, y \in \mathbf{R}^{d}}\left|\partial_{x}^{\alpha} \partial_{y}^{\beta} \omega_{j}\left(t_{j}, x, y\right)\right| \leq \kappa_{m} .
$$

(ii) Let $\left(\bar{x}_{L}, \ldots, \bar{x}_{0}\right)$ be an arbitrary solution of the system of the equation

$$
\partial_{x_{j}} S_{j+1}\left(t_{j+1}, \bar{x}_{j+1}, \bar{x}_{j}\right)+\partial_{x_{j}} S_{j}\left(t_{j}, \bar{x}_{j}, \bar{x}_{j-1}\right)=0, j=1, \ldots, L-1 .
$$

For any $m \geq 1$, there exists a constant $B_{m}$ independent of $\left(\bar{x}_{L}, \ldots, \bar{x}_{0}\right), L$ and $t_{j}, j$ $=1, \ldots, L$, but dependent on $d$ such that

$$
\sum_{j=1}^{L-1} \sum_{\substack{1 \leq|\alpha| \leq m \\|\beta|=1}}\left|\left[\left(\partial_{x_{j-1}}+\partial_{x_{j}}+\partial_{x_{j+1}}\right)^{\alpha} \partial_{x_{j}}^{\beta}\left(\omega_{j}+\omega_{j+1}\right)\right]\left(\bar{x}_{j-1}, \bar{x}_{j}, \bar{x}_{j+1}\right)\right| \leq B_{m},
$$

where $\left(\partial_{x_{j-1}}+\partial_{x_{j}}+\partial_{x_{j+1}}\right)^{\alpha}=\prod_{k=1}^{d}\left(\partial_{\left(x_{j-1}\right)_{k}}+\partial_{\left(x_{j}\right)_{k}}+\partial_{\left(x_{j+1}\right)_{k}}\right)^{\alpha_{k}}$ for a multi-index $\alpha=\left(\alpha_{1}, \ldots, \alpha_{d}\right)$.

Fujiwara's assumption for the phase function in [5] is strictly stronger than that of ours. He assumed that the phase function is of the form

$$
S\left(x_{L}, \ldots, x_{0}\right)=\sum_{j=1}^{L} S_{j}\left(t_{j}, x_{j}, x_{j-1}\right)
$$

with

$$
S_{j}\left(t_{j}, x_{j}, x_{j-1}\right)=\frac{\left|x_{j}-x_{j-1}\right|^{2}}{2 t_{j}}+t_{j} \omega_{j}\left(t_{j}, x_{j}, x_{j-1}\right), j=1, \ldots, L,
$$

where $\omega_{j}\left(t_{j}, x_{j}, x_{j-1}\right)$ satisfies the estimate (1.4). In his case, our condition (H.1)(ii) is automatically satisfied. Let $S_{j}\left(t_{j}, x_{j}, x_{j-1}\right)$ be the classical action of a charged particle moving in an electromagnetic field discussed in Yajima [9]. Then $S_{j}\left(t_{j}, x_{j}\right.$, $x_{j-1}$ ) satisfies our assumption (H.1) but does not satisfy the assumption in [5]. This will be discussed at the end of $\S 2$.

When $S\left(x_{L}, \ldots, x_{0}\right)$ satisfies (H.1), then if $T_{L}=t_{1}+\cdots+t_{L}$ is small enough, for any $x_{0}, x_{L} \in \mathbf{R}^{d}$ there exists the unique critical point $\left(x_{L-1}^{*}, \ldots, x_{1}^{*}\right)$, i.e.

$$
\partial_{x_{j}} S_{j+1}\left(t_{j+1}, x_{j+1}^{*}, x_{j}^{*}\right)+\partial_{x_{j}} S_{j}\left(t_{j}, x_{j}^{*}, x_{j-1}^{*}\right)=0, j=1, \ldots, L-1,
$$

where $x_{L}^{*}=x_{L}, x_{0}^{*}=x_{0}$ (The proof is in $\S 3$ ). 
To state the assumption for the amplitude function, we use Fujiwara's notation:

$$
a\left(x_{L}, x_{0}\right)=a\left(x_{L}, x_{L-1}^{*}, \ldots, x_{1}^{*}, x_{0}\right) .
$$

Similarly, for any pair of integers $k, m$ with $k+1<m$ let $\left(x_{k+1}^{*}, \ldots, x_{m-1}^{*}\right)$ be the partial critical point, i.e.

$$
\partial_{x_{j}} S_{\jmath+1}\left(t_{j+1}, x_{j+1}^{*}, x_{j}^{*}\right)+\partial_{x} S_{j}\left(t_{j}, x_{j}^{*}, x_{j-1}^{*}\right)=0, j=k+1, \ldots, m-1,
$$

where $x_{k}^{*}=x_{k}, x_{m}^{*}=x_{m}$. Then we set

$$
a\left(x_{L}, \ldots, x_{m}, x_{k}, \ldots, x_{0}\right)=a\left(x_{L}, \ldots, x_{m}, x_{m-1}^{*}, \ldots, x_{k+1}^{*}, x_{k}, \ldots, x_{0}\right) .
$$

If $m=k+1$, we define

$$
a\left(x_{L}, \ldots, x_{k+1}, x_{k}, \ldots, x_{0}\right)=a\left(x_{L}, \ldots, x_{k+1}, x_{k}, \ldots x_{0}\right) .
$$

The assumption for the amplitude function is the following:

(H.2) $a\left(x_{L}, \ldots, x_{0}\right)$ is a real-valued function in $\mathscr{B}\left(\mathbf{R}^{d(L+1)}\right)$. For any $K \geq 0$ there exist constants $A_{K}$ and $X_{K}$ with the following properties:

For any sequence of positive integers with $j_{0}=0<j_{1}-1<j_{1}<j_{2}-1<\cdots$ $<j_{s} \leq L, s=1, \ldots, L-1$,

$$
\left|\partial_{x_{0}}^{\alpha_{0}} \partial_{x_{L}}^{\alpha_{L}} \prod_{u=1}^{s} \partial_{x_{j u-1}}^{\alpha_{j^{-1}}} \partial_{x_{j u}}^{\alpha_{j_{u}}} a\left(\overleftarrow{x_{L}, x_{j_{s}}}, \widetilde{x_{j_{s}-1}, x_{j_{s-1}}}, \ldots, \bar{x}_{j_{1}-1}, x_{0}\right)\right| \leq A_{K} X_{K}^{s}
$$

if $\left|\alpha_{j}\right| \leq K, j=0, j_{1}-1, j_{1}, \ldots, j_{s}-1, j_{s}, L$. If $j_{s}=L$, then we read the above inequality as

$$
\left|\partial_{x_{0}}^{\alpha_{0}} \prod_{u=1}^{s} \partial_{x_{j^{-1}}}^{\alpha_{u^{-1}}} \partial_{x_{j_{u}}}^{\alpha_{j_{u}}} a\left(x_{L}, x_{j_{s}-1}, x_{j_{s-1}}, \ldots, \widetilde{x_{j_{1}-1}}, x_{0}\right)\right| \leq A_{K} X_{K}^{s}
$$

Let us state our main theorems. Let $H$ be the $d(L-1) \times d(L-1)$ matrix

$$
H=\left(\begin{array}{ccccc}
\frac{1}{t_{1}}+\frac{1}{t_{2}} & -\frac{1}{t_{2}} & 0 & 0 & \cdots \\
-\frac{1}{t_{2}} & \frac{1}{t_{2}}+\frac{1}{t_{3}} & -\frac{1}{t_{3}} & 0 & \cdots \\
0 & -\frac{1}{t_{3}} & \frac{1}{t_{3}}+\frac{1}{t_{4}} & -\frac{1}{t_{4}} & 0 \\
\vdots & \vdots & \vdots & \vdots & \vdots
\end{array}\right)
$$


and $W$ the Hessian matrix of $\sum_{j=1}^{L} \omega_{j}\left(t_{j}, x_{j}, x_{j-1}\right)$ at the critical point $\left(x_{L-1}^{*}, \ldots\right.$, $\left.x_{1}^{*}\right)$.

Theorem 1. Assume (H.1) and (H.2). There exists a positive constants $\delta$ such that if $T_{L}=t_{1}+\cdots+t_{L}<\delta$ then

$$
\begin{aligned}
& I\left(\left\{t_{j}\right\}, S, a, \nu\right)\left(x_{L}, x_{0}\right)= \\
& \left(\frac{\nu i}{2 \pi T_{L}}\right)^{d / 2} \exp \left\{-i \nu S\left(x_{L}, x_{0}\right)\right\} \operatorname{det}\left(I+H^{-1} W\right)^{-1 / 2}\left(a\left(x_{L}, x_{0}\right)+r\left(x_{L}, x_{0}\right)\right),
\end{aligned}
$$

and for any $K \geq 0$ there exist positive constants $C_{K}$ and $M(K)$ such that if $\left|\alpha_{0}\right|,\left|\alpha_{L}\right|$ $\leq K$

$$
\left|\partial_{x_{L}}^{\alpha_{L}} \partial_{x_{0}}^{\alpha_{0}} r\left(x_{L}, x_{0}\right)\right| \leq A_{M(K)}\left(\prod_{j=1}^{L}\left(1+C_{K} X_{M(K)} \nu^{-1} t_{j}\right)-1\right) .
$$

Constants $\delta$ and $C_{K}$ are independent of $a, L,\left\{t_{j}\right\}, x_{L}, x_{0}$ and $\nu$ but depend on the dimension $d$ of space $\mathbf{R}^{d}$ and $\left\{\kappa_{m}\right\}$ and $\left\{B_{m}\right\}, M(K)$ depends only on $K$ and $d$.

Theorem 2. Assume that $a \equiv 1$ and (H.1) and let $\delta$ be the constant as in Theorem 1. Then for any $K \geq 0$ there exists a constant $C_{K}$ such that if $\left|\alpha_{0}\right|,\left|\alpha_{L}\right|$ $\leq K$

$$
\left|\partial_{x_{L}}^{\alpha_{L}} \partial_{x_{0}}^{\alpha_{0}} r\left(x_{L}, x_{0}\right)\right| \leq \prod_{j=1}^{L}\left(1+C_{K} \nu^{-1} t_{j} T_{L}\right)-1
$$

We remark that our estimate of $r\left(x_{L}, x_{0}\right)$ in Theorem 1 is the same as that in Fujiwara [5], but that in Theorem 2 differs from his in the power of $T_{L}$ : our power is 1 while his power is 2 .

In $\S 2$ we see that the phase function coming from the action integral for a charged particle in an electromagnetic field satisfies (H.1). In the later sections we mimic the discussion of [5]. The existence of the critical point of the phase function is proved in $\S 3$. In $\S 4$ we write down a lemma about the stationary phase method on a space of large dimension. Theorems 1 and 2 are proved in $\S 5$.

\section{Piecewise classical path in electromagnetic fields}

We give an example of $S\left(x_{L}, \ldots, x_{0}\right)$ which satisfies the assumption (H.1). We consider a charged particle in an electromagnetic field in $\mathbf{R}^{d}$ which satisfies the assumption considered by Yajima [9]. In this section we denote the $l$-th component 
of $x \in \mathbf{R}^{d}$ by $x_{l}$. We make the following assumption for the vector and scalar potentials $A(t, x)$ and $V(x)$ :

Assumption (A). For $k=1, \ldots, d, A_{k}(t, x)$ is a real-valued function of $(t, x)$ $\in \mathbf{R} \times \mathbf{R}^{d}$, and for any $\alpha, \partial_{x}^{\alpha} A_{k}(t, x)$ is $C^{1}$ in $(t, x) \in \mathbf{R} \times \mathbf{R}^{d}$. There exists $\varepsilon>0$ such that

$$
\begin{gathered}
\left|\partial_{x}^{\alpha} A_{k}(t, x)\right|+\left|\partial_{x}^{\alpha} \partial_{t} A_{k}(t, x)\right| \leq C_{a}, \quad|\alpha| \geq 1, \quad(t, x) \in \mathbf{R} \times \mathbf{R}^{d}, \\
\left|\partial_{x}^{\alpha} B(t, x)\right| \leq C_{a}(1+|x|)^{-1-\varepsilon}, \quad|\alpha| \geq 1
\end{gathered}
$$

where $B(t, x)$ is the skew symmetric matrix with $(k, l)$-component $B_{k l}(t, x)=$ $\left(\partial A_{l} / \partial x_{k}-\partial A_{k} / \partial x_{l}\right)(t, x)$ and $|B|$ denotes the norm of matrix $B$ regarded as an operator on $\mathbf{R}^{d}, V(x)$ is a real-valued $C^{\infty}$ function which satisfies

$$
\left|\partial_{x}^{\alpha} V(x)\right| \leq C_{a}, \quad|\alpha| \geq 2 .
$$

In the form of oscillatory integrals Yajima [9] constructed the propagator for the Schrödinger evolution equation with a vector potential satisfying Assumption (A). We remark that this assumption is satisfied by constant magnetic fields.

Let $H(t, x, \xi)$ be the Hamiltonian

$$
H(t, x, \xi)=2^{-1}(\xi-A(t, x))^{2}+V(x) .
$$

Then Hamilton's differential equation is

$$
\dot{x}=\partial_{\xi} H(t, x, \xi), \quad \dot{\xi}=-\partial_{x} H(t, x, \xi)
$$

with $\dot{x}=d x / d t$ and $\dot{\xi}=d \xi / d t$. When we introduce the position-velocity variables by $(q(t), v(t))=(x(t), \xi(t)-A(t, x(t)))$, then Hamilton's differential equation is equivalent to Lagrange's differential equation:

$$
\dot{q}(t)=v(t), \quad \dot{v}(t)=B(t, q(t)) v(t)+F(t, q(t)),
$$

where $F(t, x)=-\left(\partial_{t} A\right)(t, x)-\left(\partial_{x} V\right)(x)$. The next lemma is a result of Yajima [9].

Lemma 2.1. Let $|t-s| \leq 1$.

(i) For any $\alpha$ with $|\alpha| \geq 1$, there exists a constant $C_{\alpha}^{\prime}$ such that for any solution $(q(\tau), v(\tau)), s \leq \tau \leq t$, of $(2.4)$,

$$
\int_{s}^{t}\left|\left(\partial_{x}^{\alpha} B\right)(\tau, q(\tau))\right||v(\tau)| d \tau \leq C_{\alpha}^{\prime}
$$


(ii) There exists a constant $T>0$ such that if $0<|t-s|<T$, then for any $x, y$ $\in \mathbf{R}^{d}$ there exists a unique solution $(q(\tau), v(\tau)), s \leq \tau \leq t$, of $(2.4)$ with $q(s)=y$ and $q(t)=x$.

Proof. We refer the proof to Yajima [9, Lemma 2.1 and Proposition 2.6].

Let $T>0$ be as in Lemma 2.1(ii) and $|t-s| \leq T$. We write the unique solution $q(\tau)$ of (2.4) with $q(s)=y$ and $q(t)=x$ as

$$
q(\tau)=q^{0}(\tau)+q^{1}(\tau)
$$

where $q^{0}(\tau)=\frac{\tau-s}{t-s}(x-y)+y$. Then we have

$$
\ddot{q}^{1}(\tau)=B(\tau, q(\tau)) v(\tau)+F(\tau, q(\tau))
$$

and

$$
q^{1}(s)=q^{1}(t)=0 .
$$

Let $G$ be the Green operator of the Dirichlet boundary value problem:

$$
-\ddot{q}(\tau)=f(\tau), s \leq \tau \leq t, \quad q(s)=q(t)=0 .
$$

Then we have

$$
(G f)(\tau)=\int_{s}^{t} g(\tau, u) f(u) d u
$$

where

$$
\begin{aligned}
g(\tau, u) & =\frac{(u-s)(t-\tau)}{(t-s)}, \text { if } s \leq u \leq \tau \leq t, \\
& =\frac{(\tau-s)(t-u)}{(t-s)}, \text { if } s \leq \tau \leq u \leq t
\end{aligned}
$$

Put $\|f\|_{L^{1}}=\int_{s}^{t}|f(\tau)| d \tau$ and $\|f\|_{L^{\infty}}=\sup _{s \leq \tau \leq t}|f(\tau)|$. Then we have

$$
\left\|\frac{d(G f)}{d \tau}\right\|_{L^{1}} \leq|t-s|\|f\|_{L^{1}}
$$

Lemma 2.2. There exists a constant $0<T^{0}<\min (T, 1)$ such that if $|t-s|$ $\leq T^{0}$ then for any $\alpha, \beta$ with $|\alpha+\beta| \geq 1$, 


$$
\left\|\partial_{x}^{\alpha} \partial_{y}^{\beta} q^{1}\right\|_{L^{\infty}} \leq\left\|\partial_{x}^{\alpha} \partial_{y}^{\beta} \dot{q}^{1}\right\|_{L^{1}} \leq C_{\alpha \beta}|t-s|
$$

Proof. The first inequality is Poincare's inequality. Differentiating (2.5) and using (2.6), we have

$$
\begin{aligned}
\left\|\partial_{x_{l}} \dot{q}^{1}\right\|_{L^{1}} \leq|t-s| & \| B \cdot \partial_{x_{l}}\left(\dot{q}^{0}+\dot{q}^{1}\right) \\
& \quad+\sum_{m=1}^{d} \partial_{x_{l}}\left(q^{0}+q^{1}\right)_{m} \partial_{x_{m}} B \cdot \dot{q}+\sum_{m=1}^{d} \partial_{x_{l}}\left(q^{0}+q^{1}\right)_{m} \cdot \partial_{x_{m}} F \|_{L^{1}} \\
\leq & \quad\left[t-s \mid\left[C_{1}\left(1+\left\|\partial_{x_{l}} \dot{q}^{1}\right\|_{L^{1}}\right)+C_{1}^{\prime}\left(1+\left\|\partial_{x_{l}} q^{1}\right\|_{L^{-}}\right)\right.\right. \\
& \left.\quad+C_{1}|t-s|\left(1+\left\|\partial_{x_{l}} q^{1}\right\|_{L^{2}}\right)\right] \\
\leq & |t-s|\left(C_{1}+C_{1}^{\prime}+C_{1}|t-s|\right)\left(1+\left\|\partial_{x_{l}} \dot{q}^{1}\right\|_{L^{1}}\right)
\end{aligned}
$$

noting $\partial_{x_{l}} \dot{q}_{m}^{0}=\frac{\delta_{l m}}{(t-s)}, \partial_{x_{l}} q_{m}^{0}=\frac{\delta_{l m}(\tau-s)}{(t-s)}$ and Lemma 2.1(i) and using the first inequality of (2.7). Hence if $|t-s|$ is sufficiently small, we have the second inequality of (2.7). Similar arguments lead to (2.7) for general $\alpha$ and $\beta$.

Let $S(t, s, x, y)$ be the action of the classical path $(q(\tau), v(\tau))$ joining $(s, y)$ to $(t, x)$ :

$$
S(t, s, x, y)=\int_{s}^{t} L(\tau, q(\tau), v(\tau)) d \tau
$$

where $L(\tau, q, v)$ is the Lagrangian corresponding to $H(\tau, x, \xi)$ :

$$
L(\tau, q, v)=v \xi-H(\tau, x, \xi)=\frac{v^{2}}{2}+A(\tau, q) v-V(q) .
$$

For any sequence $0=T_{0}<T_{1}<\cdots<T_{L}<T^{0}$ and any points $x^{j} \in \mathbf{R}^{d}, j=$ $0, \ldots, L$, we put

$$
S_{j}\left(t_{j}, x^{j}, x^{j-1}\right)=S\left(T_{j}, T_{j-1}, x^{j}, x^{j-1}\right), \quad j=1, \ldots, L,
$$

where $t_{j}=T_{j}-T_{j-1}$. We denote by $q_{\Delta}=q_{\Delta}^{0}+q_{\Delta}^{1}$ the piecewise classical path joining $\left(T_{j}, x^{j}\right), j=0, \ldots, L$, i.e. $q_{\Delta}^{0}$ is

$$
q_{\Delta}^{0}(\tau)=\frac{\tau-T_{j-1}}{t_{j}}\left(x^{j}-x^{j-1}\right)+x^{j-1}, \quad T_{j-1} \leq \tau \leq T_{j}, \quad j=1, \ldots, L,
$$

and $q_{\Delta}^{1}$ satisfies

$$
\ddot{q}_{\Delta}^{1}(\tau)=B\left(\tau, q_{\Delta}(\tau)\right) \dot{q}_{\Delta}(\tau)+F\left(\tau, q_{\Delta}(\tau)\right), \quad T_{j-1} \leq \tau \leq T_{j},
$$


and $q_{\Delta}^{1}\left(T_{j}\right)=0, j=0, \ldots, L$. The action along the piecewise classical path can be written as

$$
S\left(q_{\Delta}\right)=S\left(x^{L}, \ldots, x^{0}\right)=\sum_{j=1}^{L} S_{j}\left(t_{j}, x^{j}, x^{j-1}\right)
$$

THEOREM 2.3. Let $T_{L}<T^{0}$. Then $S\left(x^{L}, \ldots, x^{0}\right)=\sum_{j=1}^{L} S_{j}\left(t_{j}, x^{j}, x^{j-1}\right)$ satisfies Assumption (H.1).

Proof. First we verify (H.1). Let $q(\tau)=q^{0}(\tau)+q^{1}(\tau)$ be the classical path joining $(s, y)$ to $(t, x)$. We have

$$
\begin{aligned}
S(t, s, x, y) & =\int_{s}^{t}\left(\frac{\left|\dot{q}^{0}(\tau)+\dot{q}^{1}(\tau)\right|^{2}}{2}+A(\tau, q(\tau)) \dot{q}(\tau)-V(q(\tau))\right) d \tau \\
& =\frac{|x-y|^{2}}{2(t-s)}+\int_{s}^{t}\left(\frac{\left|\dot{q}^{1}(\tau)\right|^{2}}{2}+A(\tau, q(\tau)) \dot{q}(\tau)-V(q(\tau))\right) d \tau \\
& =\frac{|x-y|^{2}}{2(t-s)}+\omega(t, s, x, y)
\end{aligned}
$$

where

$$
\omega(t, s, x, y)=\int_{s}^{t}\left(\frac{\left|\dot{q}^{1}(\tau)\right|^{2}}{2}+A(\tau, q(\tau)) \dot{q}(\tau)-V(q(\tau))\right) d \tau
$$

Since $q$ satisfies (2.5), it follows that

$$
\left(\partial_{y_{k}} \omega\right)(t, s, x, y)=\int_{s}^{t} \partial_{y_{k}} q^{0}\left(B(\tau, q(\tau)) \dot{q}(\tau)+F(\tau, q(\tau)) d \tau-A_{k}(s, y)\right.
$$

Noting $\partial_{y_{k}} q_{m}^{0}=(t-\tau)(t-s)^{-1} \delta_{k m}$, we obtain

$$
\begin{aligned}
\left(\partial_{y_{l}} \partial_{y_{k}} \omega\right)(t, s, x, y) & =\int_{s}^{t} \frac{t-\tau}{t-s}\left(\sum_{m=1}^{d} B_{k m} \partial_{y_{l}} \dot{q}_{m}+\sum_{n, m=1}^{d} \partial_{y_{l}} q_{n} \partial_{x_{n}} B_{k m} \dot{q}_{m}\right. \\
& \left.+\sum_{m=1}^{d} \partial_{y_{l}} q_{m} \cdot \partial_{x_{m}} F_{k}\right) d \tau-\left(\partial_{y_{l}} A_{k}\right)(s, y) .
\end{aligned}
$$

So from Assumption (A), Lemma 2.1(i) and Lemma 2.2, we have

$$
\begin{array}{r}
\left|\partial_{y_{l}} \partial_{y_{h}} \omega\right| \leq C_{1}(1+C|t-s|)+C_{1}^{\prime}(1+C|t-s|)+ \\
C_{1}|t-s|(1+C|t-s|)+C_{1} \leq \kappa_{2},
\end{array}
$$

where $\kappa_{2}$ is independent of $x, y$ and $t-s$. For the other higher derivatives of $\omega$, similar arguments hold. So we have proved (H.1)(i). 
Next we show (H.1)(ii). We put

$$
\omega_{j}\left(x^{j}, x^{j-1}\right)=\omega\left(T_{j}, T_{j-1}, x^{j}, x^{j-1}\right) .
$$

In the same way as above we have

$$
\begin{aligned}
& \partial_{x_{l}^{j}} \partial_{x_{k}^{j}}\left(\omega_{j+1}\left(x^{j+1}, x^{j}\right)+\omega_{j}\left(x^{j}, x^{j-1}\right)\right) \\
& =\int_{T_{j}}^{T_{j+1}} \frac{T_{j+1}-\tau}{t_{j+1}}\left(\sum_{m=1}^{d} B_{k m} \partial_{x_{l}^{j}}\left(\dot{q}_{\Delta}^{0}+\dot{q}_{\Delta}^{1}\right)_{m}+\sum_{n, m=1}^{d} \partial_{x_{l}^{j}}\left(q_{\Delta}\right)_{n} \partial_{x_{n}} B_{k m}\left(\dot{q}_{\Delta}\right)_{m}\right. \\
& \left.\quad+\sum_{m=1}^{d} \partial_{x_{l}^{j}}\left(q_{\Delta}\right)_{m} \cdot \partial_{x_{m}} F_{k}\right) d \tau \\
& +\int_{T_{j-1}}^{T_{j}} \frac{\tau-T_{j-1}}{t_{j}}\left(\sum_{m=1}^{d} B_{k m} \partial_{x_{l}^{j}}\left(\dot{q}_{\Delta}^{0}+\dot{q}_{\Delta}^{1}\right)_{m}+\sum_{n, m=1}^{d} \partial_{x_{l}^{j}}\left(q_{\Delta}\right)_{n} \partial_{x_{n}} B_{k m}\left(\dot{q}_{\Delta}\right)_{m}\right. \\
& \left.\quad+\sum_{m=1}^{d} \partial_{x_{l}^{j}}\left(q_{\Delta}\right)_{m} \cdot \partial_{x_{m}} F_{k}\right) d \tau \\
& \partial_{x_{l}^{j+1}} \partial_{x_{k}^{j}} \omega_{j+1}\left(x^{j+1}, x^{j}\right) \\
& =\int_{T_{j}}^{T_{j+1}} \frac{T_{j+1}-\tau}{t_{j+1}}\left(\sum_{m=1}^{d} B_{k m} \partial_{x_{l}^{j+1}}\left(\dot{q}_{\Delta}^{0}+\dot{q}_{\Delta}^{1}\right)_{m}+\sum_{n, m=1}^{d} \partial_{x_{l}^{j+1}}\left(q_{\Delta}\right)_{n} \partial_{x_{n}} B_{k m}\left(\dot{q}_{\Delta}\right)_{m}\right. \\
& \left.\quad+\sum_{m=1}^{d} \partial_{x_{l}^{j+1}}\left(q_{\Delta}\right)_{m} \cdot \partial_{x_{m}} F_{k}\right) d \tau
\end{aligned}
$$

and

$$
\begin{aligned}
& \partial_{x_{l}^{j-1}} \partial_{x_{k}^{j}} \omega_{j}\left(x^{j}, x^{j-1}\right) \\
& =\int_{T_{j-1}}^{T_{j}} \frac{\tau-T_{j-1}}{t_{j}}\left(\sum_{m=1}^{d} B_{k m} \partial_{x_{l}^{j-1}}\left(\dot{q}_{\Delta}^{0}+\dot{q}_{\Delta}^{1}\right)_{m}+\sum_{n, m=1}^{d} \partial_{x_{l}^{j-1}}\left(q_{\Delta}\right)_{n} \partial_{x_{n}} B_{k m}\left(\dot{q}_{\Delta}\right)_{m}\right. \\
& \left.\quad+\sum_{m=1}^{d} \partial_{x_{l}^{j-1}}\left(q_{\Delta}\right)_{m} \cdot \partial_{x_{m}} F_{k}\right) d \tau .
\end{aligned}
$$

This together with

$$
-\partial_{x_{l}^{j}}\left(\dot{q}_{\Delta}^{0}\right)_{m}=\partial_{x_{l}^{j+1}}\left(\dot{q}_{\Delta}^{0}\right)_{m}=\frac{1}{t_{j+1}} \delta_{l m}, \quad T_{j} \leq \tau \leq T_{j+1},
$$

and

$$
-\partial_{x_{l}^{j-1}}\left(\dot{q}_{\Delta}^{0}\right)_{m}=\partial_{x_{l}^{j}}\left(\dot{q}_{\Delta}^{0}\right)_{m}=\frac{1}{t_{j}} \delta_{l m}, \quad T_{j-1} \leq \tau \leq T_{j},
$$

yields that 


$$
\begin{aligned}
& \left(\partial_{x_{l}^{j-1}}+\partial_{x_{l}^{j}}+\partial_{x_{l}^{j+1}}\right) \partial_{x_{k}^{j}}\left(\omega_{j}+\omega_{j+1}\right)\left(x^{j-1}, x^{j}, x^{j+1}\right) \\
& =\int_{T_{j}}^{T_{j+1}} \frac{T_{j+1}-\tau}{t_{j+1}}\left(\sum_{m=1}^{d} B_{k m}\left(\partial_{x_{l}^{j+1}}+\partial_{x_{l}^{j}}\right) \dot{q}_{\Delta m}^{1}\right. \\
& \left.\quad+\sum_{n, m=1}^{d}\left(\partial_{x_{l}^{j+1}}+\partial_{x_{l}^{j}}\right)\left(q_{\Delta}\right)_{n} \partial_{x_{n}} B_{k m}\left(\dot{q}_{\Delta}\right)_{m}+\sum_{m=1}^{d}\left(\partial_{x_{l}^{j+1}}+\partial_{x_{l}^{j}}\right)\left(q_{\Delta}\right)_{m} \cdot \partial_{x_{m}} F_{k}\right) d \tau \\
& \quad+\int_{T_{j-1}}^{T_{j}} \frac{\tau-T_{j-1}}{t_{j}}\left(\sum_{m=1}^{d} B_{k m}\left(\partial_{x_{l}^{j}}+\partial_{x_{l}^{j-1}}\right) \dot{q}_{\Delta m}^{1}\right. \\
& \left.\quad+\sum_{n, m=1}^{d}\left(\partial_{x_{l}^{j}}+\partial_{x_{l}^{j-1}}\right)\left(q_{\Delta}\right)_{n} \partial_{x_{n}} B_{k m}\left(\dot{q}_{\Delta}\right)_{m}+\sum_{m=1}^{d}\left(\partial_{x_{l}^{j}}+\partial_{x_{l}^{j-1}}\right)\left(q_{\Delta}\right)_{m} \cdot \partial_{x_{m}} F_{k}\right) d \tau .
\end{aligned}
$$

When $\left(\bar{x}^{L}, \ldots, \bar{x}^{0}\right)$ is a critical point of $S\left(q_{\Delta}\right)$, the piecewise classical path $q_{\Delta}(\tau)$ coincides with the classical path $q(\tau)$ joining $\left(0, \bar{x}^{0}\right)$ and $\left(T_{L}, \bar{x}^{0}\right)$. So we have from Lemma 2.2

$$
\begin{aligned}
&\left|\left(\partial_{x_{l}^{j-1}}+\partial_{x_{l}^{j}}+\partial_{x_{l}^{j+1}}\right) \partial_{x_{k}^{j}}\left(\omega_{j}+\omega_{j+1}\right)\left(\bar{x}^{j-1}, \bar{x}^{j}, x^{j+1}\right)\right| \\
& \leq C\left(t_{j+1}+t_{j}\right)+C \int_{T_{j-1}}^{T_{j+1}}|(\partial B)(\tau, q(\tau))||v(\tau)| d \tau .
\end{aligned}
$$

Therefore, we have by Lemma 2.1(i)

$$
\begin{aligned}
& \sum_{j=1}^{L-1}\left|\left(\partial_{x_{l}^{j-1}}+\partial_{x_{k}^{j}}+\partial_{x_{l}^{j+1}}\right) \partial_{x_{k}^{j}}\left(\omega_{j}+\omega_{j+1}\right)\left(\bar{x}^{j-1}, \bar{x}^{j}, \bar{x}^{j+1}\right)\right| \\
& \quad \leq C T_{L}+C \int_{0}^{T_{L}}|(\partial B)(\tau, q(\tau))||v(\tau)| d \tau \\
& \quad \leq B_{1},
\end{aligned}
$$

where $B_{1}$ is independent of $\left(\bar{x}^{L}, \ldots, \bar{x}^{0}\right), L$ and $T_{L}$ if $T_{L}<T^{0}$. Similar discussions hold for other differentiation $\left(\partial_{x^{j-1}}+\partial_{x^{j}}+\partial_{x^{j+1}}\right)^{\alpha}$. Thus we have proved (H.1)(ii).

Finally we remark that our phase function $S(t, s, x, y)$ does not satisfy Fujiwara's assumption in [5]. In fact, in the case that $V(x) \equiv 0$ and $A(t, x)=$ $A^{0} x$, where $A^{0}$ is a real constant $d \times d$ matrix, we can see from (2.10) that

$$
\begin{aligned}
\left(\partial_{y_{l}} \partial_{y_{k}} \omega\right)(t, s, x, y) & =\int_{s}^{t} \frac{t-\tau}{t-s}\left(\sum_{m=1}^{d} B_{k m} \partial_{y_{l}} \dot{q}_{m}\right) d \tau-\left(\partial_{y_{l}} A_{k}\right)(s, y) \\
& =-\frac{A_{k l}^{0}+A_{l k}^{0}}{2}+\int_{s}^{t} \frac{t-\tau}{t-s}\left(\sum_{m=1}^{d} B_{k m} \partial_{y_{l}} \dot{q}_{m}^{1}\right) d \tau
\end{aligned}
$$




$$
=-\frac{A_{k l}^{0}+A_{l k}^{0}}{2}+O(t-s)
$$

as $t-s$ goes to zero.

\section{Phase functions}

In this section we discuss the unique existence of the critical point of $S$ (Lemma 3.5) and study some of its properties. The method is similar to that of Yajima [9]. In what follows, we assume (H.1) and abbreviate $S_{j}\left(t_{j}, x_{j}, x_{j-1}\right)$ as $S_{j}\left(x_{j}, x_{j-1}\right)$ and $\omega_{j}\left(t_{j}, x_{j}, x_{j-1}\right)$ as $\omega_{j}\left(x_{j}, x_{j-1}\right)$. To avoid additional complexity we put $d=1$.

Lemma 3.1. Let $2 t_{j} \kappa_{2} \leq 1, j=1, \ldots, L$. Then for any $y$ and $k \in \mathbf{R}$, there exists a unique $\left(x_{0}^{\#}, \ldots, x_{L}^{\#}\right)=\left(x_{0}^{\#}(y, k), \ldots, x_{L}^{\#}(y, k)\right)$ which satisfies $x_{0}^{\#}=y$, $\frac{x_{1}^{\#}-y}{t_{1}}=k$ and

$$
\frac{x_{j+1}^{\#}-x_{j}^{\#}}{t_{j+1}}-\frac{x_{j}^{\#}-x_{j-1}^{\#}}{t_{j}}=\partial_{j} \omega_{j}\left(x_{j}^{\#}, x_{j-1}^{\#}\right)+\partial_{j} \omega_{j+1}\left(x_{j+1}^{\#}, x_{j}^{\#}\right), \quad j=1, \ldots, L-1 .
$$

Proof. We have $x_{1}^{\#}=x_{1}^{\#}(y, k)=t_{1} k+y$. Put

$$
k_{j}^{\#}=\frac{x_{j}^{\#}-x_{j-1}^{\#}}{t_{j}}, j=1, \ldots, L \text {. }
$$

Then the system of the equation (3.1) is equivalent to

$$
\begin{aligned}
& k_{j+1}^{\#}-k_{j}^{\#}=\partial_{j} \omega_{j}\left(x_{j-1}^{\#}+t_{j} k_{j}^{\#}, x_{j-1}^{\#}\right) \\
& +\partial_{j} \omega_{j+1}\left(x_{j-1}^{\#}+t_{j} k_{j}^{\#}+t_{j+1} k_{j+1}^{\#}, x_{j-1}^{\#}+t_{j} k_{j}^{\#}\right), \quad j=1, \ldots, L-1 .
\end{aligned}
$$

If $2 t_{2} \kappa_{2} \leq 1$, for any $y, k \in \mathbf{R}$, the map $\Phi_{1}$ :

$$
k_{2} \mapsto \Phi_{1}\left(k_{2}\right)=k+\left(\partial_{1} \omega_{1}\right)\left(y+t_{1} k, y\right)+\left(\partial_{1} \omega_{2}\right)\left(y+t_{1} k+t_{2} k_{2}, y+t_{1} k\right)
$$

is a contraction. So there exists a unique $k_{2}^{\#}=k_{2}^{\#}(y, k)$ which satisfies (3.3) for $j=1$. Hence we have $x_{2}^{\#}(y, k)=x_{1}^{\#}(y, k)+t_{2} k_{2}^{\#}(y, k)$. Similarly we have the unique existence of $k_{3}^{\#}, \ldots, k_{L}^{\#}$ and $x_{3}^{\#}, \ldots, x_{L}^{\#}$, successively.

As in the proof of Lemma 3.1 , we set $k_{j}^{\#}(y, k)=\frac{x_{j}^{\#}(y, k)-x_{j-1}^{\#}(y, k)}{t_{j}}$, $j=1, \ldots, L$, where $k_{1}^{\#}=k$ and $x_{0}^{\#}=y$. Let $T_{j}=t_{1}+\cdots+t_{j}$. 
Lemma 3.2. If $2 t_{j} \kappa_{2} \leq 1, j=1, \ldots, L$, then for $|\alpha+\beta| \geq 1$,

$$
\begin{gathered}
\left|\partial_{y}^{\alpha} \partial_{k}^{\beta}\left(x_{j}^{\#}(y, k)-y-T_{j} k\right)\right| \leq C_{\alpha \beta} T_{j}^{|\beta|+1}, \\
\left|\partial_{y}^{\alpha} \partial_{k}^{\beta}\left(k_{j}^{\#}(y, k)-k\right)\right| \leq C_{\alpha \beta} T_{j}^{|\beta|} .
\end{gathered}
$$

Proof. We prove this by induction on $l=|\alpha+\beta|$. We denote $x_{j}^{\#}(y, k)$ by $x_{j}, k_{j}^{\#}(y, k)$ by $k_{j}, \partial_{y}^{\alpha} \partial_{k}^{\beta} x_{j}^{\#}$ by $x_{j}^{\alpha \beta}$ and $\partial_{y}^{\alpha} \partial_{k}^{\beta} k_{j}^{\#}$ by $k_{j}^{\alpha \beta}$.

Let $l=1$. Then we have from $(3.2,3)$,

$$
\begin{aligned}
& x_{j}^{\alpha \beta}-x_{j-1}^{\alpha \beta}=t_{j} k_{j}^{\alpha \beta}, \quad j=1, \ldots, L, \\
& k_{j+1}^{\alpha \beta}-k_{j}^{\alpha \beta}=\left(\partial_{j-1}+\partial_{j}+\partial_{j+1}\right) \partial_{j}\left(\omega_{j}+\omega_{j+1}\right) x_{j-1}^{\alpha \beta} \\
& +\left(\partial_{j}^{2}\left(\omega_{j}+\omega_{j+1}\right)+\partial_{j+1} \partial_{j} \omega_{j+1}\right) t_{j} k_{j}^{\alpha \beta}+\partial_{j+1} \partial_{j} \omega_{j+1} t_{j+1} k_{j+1}^{\alpha \beta}, \quad j=1, \ldots, L-1 .
\end{aligned}
$$

So we obtain with $\phi_{j}=\left(\partial_{j-1}+\partial_{j}+\partial_{j+1}\right) \partial_{j}\left(\omega_{j}+\omega_{j+1}\right)\left(x_{j-1}, x_{j}, x_{j+1}\right)$

$$
\left(1-\kappa_{2} t_{j+1}\right)\left|k_{j+1}^{\alpha \beta}\right|+\left|x_{j}^{\alpha \beta}\right| \leq\left(1+\left(3 \kappa_{2}+1\right) t_{j}\right)\left|k_{j}^{\alpha \beta}\right|+\left(1+\left|\phi_{j}\right|\right)\left|x_{j-1}^{\alpha \beta}\right| .
$$

Hence if $1-\kappa_{2} t_{j+1} \geq 1 / 2$, then

$$
\left|k_{j+1}^{\alpha \beta}\right|+\left|x_{j}^{\alpha \beta}\right| \leq\left(1+2\left|\phi_{j}\right|+2\left(3 \kappa_{2}+1\right) t_{j}+2 \kappa_{2} t_{j+1}\right)\left(\left|k_{j}^{\alpha \beta}\right|+\left|x_{j-1}^{\alpha \beta}\right|\right) .
$$

Here we have used $(1+b)(1-a)^{-1} \leq 1+2(a+b)$ for $0 \leq 2 a \leq 1$. Since $k_{1}^{\alpha \beta}$, $x_{0}^{\alpha \beta}=0$ or 1 , it follows from Assumption (H.1)(ii) that $\left|k_{j+1}^{\alpha \beta}\right|+\left|x_{j}^{\alpha \beta}\right| \leq C$. So we have

$$
\left|\partial_{y}\left(k_{j}-k\right)\right| \leq C \text { and }\left|\partial_{y}\left(x_{j}-y-T_{j} k\right)\right|=\left|\sum_{l=1}^{j} t_{l} \partial_{y} k_{l}\right| \leq C T_{j} .
$$

Moreover since we have

$$
\left|\partial_{k} x_{j}\right|=\left|\partial_{k}\left(x_{j}-y\right)\right|=\left|\sum_{l=1}^{j} t_{l} \partial_{y} k_{l}\right| \leq C T_{j},
$$

we obtain by summing (3.6) for $j$

$$
\left|\partial_{k}\left(k_{j}-k\right)\right| \leq C T_{j} \text { and }\left|\partial_{k}\left(x_{j}-y-T_{j} k\right)\right|=\left|\sum_{l=1}^{j} t_{l} \partial_{k}\left(k_{l}-k\right)\right| \leq C T_{j}^{2} .
$$

Next we suppose that $(3.4,5)$ are true for $|\alpha+\beta| \leq l$ and prove them for $|\alpha+\beta|=l+1$. We put

$$
\begin{aligned}
& g\left(x_{j-1}, k_{j}, k_{j+1}\right) \\
& =\left(\partial_{j} \omega_{j}\right)\left(x_{j-1}+t_{j} k_{j}, x_{j-1}\right)+\left(\partial_{j} \omega_{j+1}\right)\left(x_{j-1}+t_{j} k_{j}+t_{j+1} k_{j+1}, x_{j-1}+t_{j} k_{j}\right) .
\end{aligned}
$$


Differentiating (3.3) we have

$$
\begin{aligned}
& k_{j+1}^{\alpha \beta}-k_{j}^{\alpha \beta}=\partial_{x_{j-1}} g \cdot x_{j-1}^{\alpha \beta}+\partial_{k_{j}} g \cdot k_{j}^{\alpha \beta}+\partial_{k_{j+1}} g \cdot k_{j+1}^{\alpha \beta} \\
& +\sum C \partial_{x_{j-1}}^{r} g \cdot x_{j-1}^{\bar{\alpha}_{1} \bar{\beta}_{1}} \cdots x_{j-1}^{\bar{\alpha}_{i, \beta} \overline{\beta_{1+}}}
\end{aligned}
$$

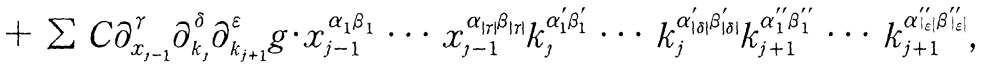

where the sums are taken in the suitable manner, and

$$
\begin{gathered}
\left(\bar{\alpha}_{1}, \bar{\beta}_{1}\right)+\cdots+\left(\bar{\alpha}_{|\gamma|}, \bar{\beta}_{|\gamma|}\right)=(\alpha, \beta), \quad 2 \leq|\gamma| \leq l+1 \\
\left(\alpha_{1}, \beta_{1}\right)+\cdots+\left(\alpha_{|\gamma|}, \beta_{\mid \gamma}\right)+\left(\alpha_{1}^{\prime}, \beta_{1}^{\prime}\right)+\cdots+\left(\alpha_{|\delta|}^{\prime}, \beta_{|\delta|}^{\prime}\right) \\
+\left(\alpha_{1}^{\prime \prime}, \beta_{1}^{\prime \prime}\right)+\cdots+\left(\alpha_{\mid \varepsilon}^{\prime \prime}, \beta_{|\varepsilon|}^{\prime \prime}\right)=(\alpha, \beta) \\
2 \leq|\gamma|+|\delta|+|\varepsilon| \leq l+1 \text { and } 1 \leq|\delta|+|\varepsilon|
\end{gathered}
$$

It is clear that

$$
\begin{aligned}
\partial_{x_{j-1}} g & =\left(\partial_{,-1}+\partial_{j}+\partial_{j+1}\right) \partial_{j}\left(\omega_{j}+\omega_{j+1}\right), \\
\partial_{k j} g & =t,\left(\partial_{j}^{2}\left(\omega_{j}+\omega_{j+1}\right)+\partial_{j+1} \partial_{,} \omega_{j+1}\right), \partial_{k_{j+1}} g=t_{j+1} \partial_{\jmath+1} \partial_{\jmath} \omega_{\jmath+1}, \\
\partial_{x_{j-1}}^{r} g & =\left(\partial_{\jmath-1}+\partial_{\jmath}+\partial_{\jmath+1}\right)^{r} \partial_{\jmath}\left(\omega_{j}+\omega_{j+1}\right) \\
\quad \text { and } & \left|\partial_{x_{j-1}}^{r} \partial_{k_{j}}^{\delta} \partial_{k_{j+1}}^{\varepsilon} g\right| \leq C_{\alpha \beta} \kappa_{l+1} t_{j}^{|\delta|} t_{j+1}^{|\varepsilon|} .
\end{aligned}
$$

By induction hypothesis (3.4) we have

$$
\left|x_{j-1}^{\bar{\alpha}_{1} \bar{\beta}_{1}} \cdots x_{j-1}^{\bar{\alpha}_{|n| \bar{\beta}|\lambda|}}\right| \leq C_{\alpha \beta} T_{j-1}^{|\beta|} \text {. }
$$

We can show that

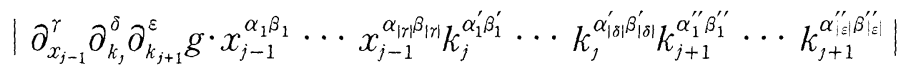

$$
\begin{aligned}
& \leq C_{\alpha \beta}\left(t_{j}+t_{j+1}\right) T_{j+1}^{(|\beta|-1)_{+}},
\end{aligned}
$$

with $(a)_{+}=\max (a, 0)$. In fact, in the case $0 \leq|\beta|-\left|\beta_{1}+\cdots+\beta_{|r|}\right| \leq 1$, it is clear from $|\delta+\varepsilon| \geq 1$. In the case that $|\beta|-\left|\beta_{1}+\cdots+\beta_{|\gamma|}\right|=s \geq 2$, if $s \leq|\delta+\varepsilon|$, then the left-hand side of (3.7) is less than or equal to

$$
C_{\alpha \beta}\left(t_{j}+t_{j+1}\right)^{s} T_{j-1}^{\left|\beta_{1}+\cdots+\beta_{|x|}\right|} \leq C_{\alpha \beta}\left(t_{j}+t_{j+1}\right) T_{j+1}^{|\beta|-1} .
$$

If $s>|\delta+\varepsilon|$, then the left-hand side of (3.7) is less than or equal to

$$
C_{\alpha \beta}\left(t_{j}+t_{j+1}\right)^{|\hat{\delta}+\varepsilon|} T_{j-1}^{\left|\beta_{1}+\cdots+\beta_{|x|}\right|} T_{j+1}^{\sigma} \leq C_{\alpha \beta}\left(t_{j}+t_{j+1}\right) T_{j+1}^{|\beta|},
$$

with $\sigma=\sum_{\left|\beta_{m}^{\prime}\right| \geq 2}\left|\beta_{m}^{\prime}\right|+\sum_{\mid \beta_{m}^{\prime \prime} \geq 2}\left|\beta_{m}^{\prime \prime}\right|$, because $|\delta+\varepsilon|-1 \geq \sum_{\left|\beta_{m}^{\prime}\right|=1}\left|\beta_{m}^{\prime}\right|+$ $\sum_{\left|\beta_{m}^{\prime \prime}\right|=1}\left|\beta_{m}^{\prime \prime}\right|$. So we have together with $x_{j}^{\alpha \beta}-x_{j-1}^{\alpha \beta}=t_{j} k_{j}^{\alpha \beta}$,

$$
\begin{aligned}
\left(1-\kappa_{2} t_{j+1}\right)\left|k_{j+1}^{\alpha \beta}\right|+\left|x_{j}^{\alpha \beta}\right| & \leq\left(1+\left(2 \kappa_{2}+1\right) t_{j}\right)\left|k_{j}^{\alpha \beta}\right|+\left(1+\left|\phi_{j}\right|\right)\left|x_{j-1}^{\alpha \beta}\right| \\
& +C_{\alpha \beta}\left|\phi_{j}^{(l+1)}\right| T_{j+1}^{|\beta|}+C_{\alpha \beta}\left(t_{j}+t_{j+1}\right) T_{j+1}^{(\mid \beta !-1)_{+}},
\end{aligned}
$$


where $\phi_{j}^{(l+1)}\left(x_{j-1}, x_{j}, x_{j+1}\right)=\sum_{1 \leq|\alpha| \leq l+1} \mid\left(\partial_{j-1}+\partial_{j}+\partial_{j+1}\right)^{\alpha} \partial_{j}\left(\omega_{j}+\omega_{j+1}\right)\left(x_{j-1}\right.$, $\left.x_{j}, x_{j+1}\right) \mid$. Hence if $1-\kappa_{2} t_{j+1} \geq 1 / 2$, then

$$
\begin{aligned}
\left|k_{j+1}^{\alpha \beta}\right|+\left|x_{j}^{\alpha \beta}\right| \leq & \left(1+2\left|\phi_{j}\right|+2\left(2 \kappa_{2}+1\right) t_{j}+2 \kappa_{2} t_{j+1}\right)\left(\left|k_{j}^{\alpha \beta}\right|+\left|x_{j-1}^{\alpha \beta}\right|\right) \\
& +2 C_{\alpha \beta}\left(\left|\phi_{j}^{(l+1)}\right| T_{j+1}^{|\beta|}+\left(t_{j}+t_{j+1}\right) T_{j+1}^{(|\beta|-1)_{+}}\right) .
\end{aligned}
$$

It follows from Assumption (H.1)(ii) and $x_{0}^{\alpha \beta}=k_{1}^{\alpha \beta}=0$ that

$$
\left|k_{j+1}^{\alpha \beta}\right|+\left|x_{j}^{\alpha \beta}\right| \leq C_{\alpha \beta} T_{j+1}^{|\beta|} \text {. }
$$

Hence we have

$$
\left|k_{j}^{\alpha \beta}\right| \leq C_{\alpha \beta} T_{j}^{|\beta|} \text { and }\left|x_{j}^{\alpha \beta}\right|=\left|\sum_{l=1}^{j} t_{l} k_{l}^{\alpha \beta}\right| \leq C_{\alpha \beta} T_{j}^{|\beta|+1}
$$

The proof is completed.

We need the inverse of the map $(y, k) \mapsto\left(y, x_{L}^{\#}(y, k)\right)$. To this end we introduce the new variables:

$$
\tilde{x}(y, k)=x_{j}^{\#}\left(y, k / T_{j}\right) \text { and } \tilde{k}_{j}(y, k)=T, k_{j}^{\#}\left(y, k / T_{j}\right), \quad j=1, \ldots, L .
$$

Lemma 3.3. For any $\alpha$ and $\beta$, there exists $C_{\alpha \beta}$ such that

$$
\begin{aligned}
& \left|\partial_{y}^{\alpha} \partial_{k}^{\beta}\left(\partial_{y} \tilde{x}_{j}-1\right)\right|+\left|\partial_{y}^{\alpha} \partial_{k}^{\beta}\left(\partial_{k} \tilde{x}_{j}-1\right)\right| \\
& +\left|\partial_{y}^{\alpha} \partial_{k}^{\beta}\left(\partial_{y} \tilde{x}_{j}\right)\right|+\left|\partial_{y}^{\alpha} \partial_{k}^{\beta}\left(\partial_{k} \tilde{k}_{j}-1\right)\right| \leq C_{\alpha \beta} T_{j} .
\end{aligned}
$$

Proof. This follows from Lemma 3.2.

Lemma 3.4. There exists a constant $T>0$ such that if $T_{L}<T$, then the map $(y, k) \mapsto(y, x)=\left(y, \tilde{x}_{L}(y, k)\right)$ is a global diffeomorphism on $\mathbf{R} \times \mathbf{R}$.

Proof. Let $T$ satisfy $2 C_{00} T \leq 1$ with the constant $C_{00}$ in Lemma 3.3 and $2 \kappa_{2} T$ $\leq 1$. Then by Lemma 3.3 the map $k \mapsto U(k)=x+k-\tilde{x}_{L}(y, k)$ is a contraction. So Lemma 3.4 is proved.

Let $(y, \tilde{k}(y, x))$ be the inverse of the map $(y, k) \mapsto(y, x)=\left(y, \tilde{x}_{L}(y, k)\right)$ in Lemma 3.4 and set $k(y, x)=\tilde{k}(y, x) / T_{L}$. Put

$$
\begin{aligned}
& x_{j}^{*}(y, x)=x_{j}^{\#}(y, k(y, x)), j=1, \ldots, L-1, \\
& k_{j}^{*}(y, x)=\frac{x_{j}^{*}(y, x)-x_{j-1}^{*}(y, x)}{t_{j}}, j=1, \ldots, L,
\end{aligned}
$$


where $x_{0}^{*}=y$ and $x_{L}^{*}=x$.

Lemma 3.5. If $T_{L}<T$, then $x_{j}^{*}(y, x), j=1, \ldots, L-1$ is the unique critical point of $S$ with $x_{0}^{*}=y$ and $x_{L}^{*}=x$, i.e. it satisfies (1.7).

Proof. Let $y, x \in \mathbf{R}$. Then by Lemma 3.1, for $y, k=k(y, x)$ there exists a unique $\left(x_{0}^{\#}(y, k), \ldots, x_{L}^{\#}(y, k)\right)$ which satisfies (3.1). And we have $x_{L}^{\#}(y, k(y, x))$ $=x$ by Lemma 3.4. These $x_{j}^{\#}(y, k(y, x))$ are nothing but the desired $x_{j}^{*}(y, x)$.

The next lemma gives the estimates of the critical point.

LEMma 3.6. We have

$$
\begin{gathered}
\left|T_{L} \partial_{y} k_{j}^{*}+1\right|+\left|T_{L} \partial_{x} k_{j}^{*}-1\right| \leq C T_{L}, \quad 1 \leq j \leq L . \\
\left|\partial_{y}^{\alpha} \partial_{x}^{\beta} k_{j}^{*}\right| \leq C_{\alpha \beta}, \quad|\alpha+\beta| \geq 2, \quad 1 \leq j \leq L . \\
\left|\partial_{y} x_{j}^{*}\right|+\left|\partial_{x} x_{j}^{*}\right| \leq C, \quad 1 \leq j \leq L-1 . \\
\left|\partial_{y}^{\alpha} \partial_{x}^{\beta} x_{j}^{*}\right| \leq C_{\alpha \beta} T_{L}, \quad|\alpha+\beta| \geq 2, \quad 1 \leq j \leq L-1 .
\end{gathered}
$$

Proof. (3.10): From the facts that $T_{L} k_{1}^{*}(y, x)=T_{L} k(y, x)=\tilde{k}(y, x)$ and

$$
\tilde{x}_{L}(y, \tilde{k}(y, x))=x,
$$

differentiating (3.14) and using Lemma 3.3 we have (3.10) for the case $j=1$.

(3.10) for $2 \leq j \leq L$ follow from Lemma 3.3, (3.10) for $j=1$ and from the fact that

$$
T_{j} k_{j}^{*}(y, x)=T_{j} k_{j}^{\#}(y, k(y, x))=\tilde{k}_{j}\left(y, \frac{T_{j}}{T_{L}} \tilde{k}(y, x)\right) .
$$

(3.11): For $|\alpha+\beta| \geq 2$, differentiating (3.14) we have

$$
\begin{aligned}
0=\partial_{y}^{\alpha} \partial_{x}^{\beta} \tilde{x}_{L}(y, \tilde{k}(y, x))= & \partial_{k} \tilde{x}_{L} \cdot \partial_{y}^{\alpha} \partial_{x}^{\beta} \tilde{k} \\
& +\sum C \partial_{y}^{\alpha^{\prime}} \partial_{k}^{\beta^{\prime}} \tilde{x}_{L} \cdot \partial_{y}^{\alpha_{1}} \partial_{x}^{\beta_{1}} \tilde{k} \cdots \partial_{y}^{\alpha_{l}} \partial_{x}^{\beta_{l}} \tilde{k},
\end{aligned}
$$

where $2 \leq\left|\alpha^{\prime}+\beta^{\prime}\right|, 0 \leq l \leq|\alpha+\beta|,(0,0) \neq\left(\alpha_{m}, \beta_{m}\right)<(\alpha, \beta), 1 \leq m \leq l$ and $\left(\alpha_{1}, \beta_{1}\right)+\cdots+\left(\alpha_{l}, \beta_{l}\right) \leq(\alpha, \beta)$. Hence we have (3.11) for the case $j=1$ :

$$
\left|\left(\partial_{y}^{\alpha} \partial_{x}^{\beta} \tilde{k}\right)(y, x)\right| \leq C_{\alpha \beta} T_{L}
$$

by induction on $n=|\alpha+\beta|$, using Lemma 3.3.

Similarly, differentiating (3.15) we have 


$$
\left|\left(\partial_{y}^{\alpha} \partial_{x}^{\beta} T_{j} k_{j}^{*}\right)(y, x)\right| \leq C_{\alpha \beta} T_{3}
$$

by induction, using Lemma 3.3 and the estimate for $\partial_{y}^{\alpha} \partial_{x}^{\beta} \tilde{k}(y, x)$.

(3.12): Since we have

$$
x_{j}^{*}(y, x)=x_{j}^{\#}(y, k(y, x))=\tilde{x}_{j}\left(y, \frac{T_{j}}{T_{L}} \tilde{k}(y, x)\right),
$$

the proof is clear by (3.10) and Lemma 3.3.

(3.13): For $|\alpha+\beta| \geq 2$, we have similarly to the proof of (3.11)

$$
\begin{aligned}
\partial_{y}^{\alpha} \partial_{x}^{\beta} x_{j}^{*}(y, x)= & \partial_{k} \tilde{x}_{j} \cdot\left(T_{j} / T_{L}\right) \partial_{y}^{\alpha} \partial_{x}^{\beta} \tilde{k} \\
& +\sum C \partial_{y}^{\alpha^{\prime}} \partial_{k}^{\beta^{\prime}} \tilde{x}_{j} \cdot\left(T_{j} / T_{L}\right) \partial_{y}^{\alpha_{1}} \partial_{x}^{\beta_{1}} \tilde{k} \cdots\left(T_{j} / T_{L}\right) \partial_{y}^{\alpha_{l}} \partial_{x}^{\beta_{l}} \tilde{k},
\end{aligned}
$$

where $2 \leq\left|\alpha^{\prime}+\beta^{\prime}\right|, 0 \leq l \leq|\alpha+\beta|,(0,0) \neq\left(\alpha_{m}, \beta_{m}\right)<(\alpha, \beta), 1 \leq m \leq l$ and $\left(\alpha_{1}, \beta_{1}\right)+\cdots+\left(\alpha_{l}, \beta_{l}\right) \leq(\alpha, \beta)$. Therefore from (3.11) and Lemma 3.3, we have (3.13).

We introduce the same notations as in [5]. Let $m$ and $k$ be two positive integers with $m>k+1$. We define $\left(x_{m-1}^{*}, \ldots, x_{k+1}^{*}\right)$ as the partial critical point, i.e.

$$
\partial_{j} S_{j+1}\left(x_{j+1}^{*}, x_{j}^{*}\right)+\partial_{j} S_{j}\left(x_{j}^{*}, x_{j-1}^{*}\right)=0, j=k+1, \ldots, m-1 .
$$

Here $x_{m}^{*}=x_{m}$ and $x_{k}^{*}=x_{k}$. We denote the critical level by $S_{m, k+1}^{\#}\left(x_{m}, x_{k}\right)$, i.e.

$$
S_{m, k+1}^{\#}\left(x_{m}, x_{k}\right)=S_{m}\left(x_{m}, x_{m-1}^{*}\right)+\cdots+S_{k+1}\left(x_{k+1}^{*}, x_{k}\right) .
$$

If $k+1=m$, then we set $S_{m, k+1}^{\#}\left(x_{m}, x_{k}\right)=S_{m}\left(x_{m}, x_{m-1}\right)$. For any $m>k$, we put $T(m, k)=t_{m}+\cdots+t_{k}$, and $T(k, k)=t_{k}$. For a sequence of integers $\left(j_{1}, \ldots, j_{s}\right)$ such as $0=j_{0}<j_{1}<j_{2}<\cdots<j_{s}<L=j_{s+1}$, we put

$$
S_{j_{s} \cdots j_{1}}^{\#}\left(x_{L}, x_{j_{s}}, \ldots, x_{j_{1}}, x_{0}\right)=\sum_{r=1}^{s+1} S_{j_{r, y_{r-1}+1}}^{\#}\left(x_{j_{r}}, x_{j_{r-1}}\right) .
$$

Lemma 3.7. Let $T_{L}<T$. Then $S_{j_{s} \cdots j_{1}}^{\#}\left(x_{L}, x_{j_{s}}, \ldots, x_{j_{1}}, x_{0}\right)$ satisfies (H.1) with constants $\kappa_{m}^{\#}$ and $B_{m}^{\#}$ different from $\kappa_{m}$ and $B_{m}$ :

(i') $S_{j_{r}, j_{r-1}+1}^{\#}\left(x_{j_{r}}, x_{j_{r-1}}\right)$ is of the form

$$
S_{j_{r, j_{r-1}+1}}^{\#}\left(x_{j_{r}}, x_{j_{r-1}}\right)=\frac{\left|x_{j_{r}}-x_{j_{r-1}}\right|^{2}}{2 T\left(j_{r}, j_{r-1}+1\right)}+\omega_{j_{r, j} j_{r-1}+1}^{\#}\left(x_{j_{r}}, x_{j_{r-1}}\right) .
$$

For any $m \geq 2$, there exists $\kappa_{m}^{\#}$ such that

$$
\max _{2 \leq|\alpha+\beta| \leq m} \sup _{x, y}\left|\partial_{x}^{\alpha} \partial_{y}^{\beta} \omega_{j_{r}, j_{r-1}+1}^{\#}(x, y)\right| \leq \kappa_{m}^{\#},
$$


where $\kappa_{m}^{\#}$ depends on $\left\{\kappa_{l}\right\}$ and $\left\{B_{l}\right\}$ but not on $r$ and $t_{j}$.

(ii') Let $\left(\bar{x}_{L}, \bar{x}_{j_{s}}, \ldots, \bar{x}_{j_{1}}, \bar{x}_{0}\right)$ be an arbitrary critical point of $S_{j_{s} \cdots j_{1}}^{\#}$, i.e.

$$
\partial_{j_{r}} S_{j_{r+1}, j_{r}+1}^{\#}\left(\bar{x}_{j_{r+1}}, \bar{x}_{j_{r}}\right)+\partial_{j_{r}} S_{j_{r} j_{r-1}+1}^{\#}\left(\bar{x}_{j_{r}}, \bar{x}_{j_{r-1}}\right)=0, \quad r=1, \ldots, s .
$$

Then for any $K \geq 1$ there exists $B_{K}^{\#}$ such that

$$
\begin{gathered}
\sum_{r=1|\beta|=1,1 \leq|\alpha| \leq K}^{s}\left|\left[\left(\partial_{j_{r-1}}+\partial_{j_{r}}+\partial_{j_{r+1}}\right)^{\alpha} \partial_{j_{r}}^{\beta}\left(\omega_{j_{r} j_{r-1}+1}^{\#}+\omega_{j_{r+1}, j_{r}+1}^{\#}\right)\right]\left(\bar{x}_{j_{r-1}}, \bar{x}_{j_{r}}, \bar{x}_{j_{r+1}}\right)\right| \\
\leq B_{K}^{\#},
\end{gathered}
$$

where $B_{K}^{\#}$ depends on $\left\{\kappa_{l}\right\}$ and $\left\{B_{l}\right\}$ but not on $\left(\bar{x}_{L}, \bar{x}_{j_{s}}, \ldots, \bar{x}_{j_{1}}, \bar{x}_{0}\right)$ and $s$.

Proof. (i') We investigate simply $S\left(\widetilde{x_{L}, x_{0}}\right)$ instead of $S_{j_{r}, j_{r-1}+1}^{\#}\left(x_{j_{r}}, x_{j_{r-1}}\right)$, to which a similar argument applies. Since $\left(x_{L-1}^{*}, \ldots, x_{1}^{*}\right)$ is the critical point of $S$, we have

$$
\begin{aligned}
\partial_{0} S\left(x_{L}, x_{0}\right. & =\partial_{0}\left(S\left(x_{L}, x_{L-1}^{*}, \ldots, x_{1}^{*}, x_{0}\right)\right) \\
& =\left(\partial_{0} S_{1}\right)\left(x_{1}^{*}, x_{0}\right) .
\end{aligned}
$$

Hence we have

$$
\begin{aligned}
\partial_{0}^{2} S\left(\widetilde{x_{L}, x_{0}}\right) & =\left(\partial_{0}^{2} S_{1}\right)\left(x_{1}^{*}, x_{0}\right)+\left(\partial_{1} \partial_{0} S_{1}\right)\left(x_{1}^{*}, x_{0}\right) \partial_{0} x_{1}^{*} \\
& =t_{1}^{-1}+\partial_{0}^{2} \omega_{1}+\left(-t_{1}^{-1}+\partial_{1} \partial_{0} \omega_{1}\right)\left(1+t_{1} \partial_{0} k_{1}^{*}\right) \\
& =\partial_{0}^{2} \omega_{1}+\partial_{1} \partial_{0} \omega_{1}+\left(-1+t_{1} \partial_{0} \partial_{1} \omega_{1}\right) \partial_{0} k_{1}^{*},
\end{aligned}
$$

where we have used $\partial_{0} x_{1}^{*}=1+t_{1} \partial_{0} k_{1}^{*}$ which follows from (3.9). Since by $(3.10,11)$ of Lemma 3.6 we can write

$$
\partial_{0} k_{1}^{*}\left(x_{L}, x_{0}\right)=-\frac{1}{T_{L}}+b\left(x_{L}, x_{0}\right), \quad b\left(x_{L}, x_{0}\right) \in \mathscr{B}(\mathbf{R} \times \mathbf{R}),
$$

we have

$$
\partial_{0}^{2} S\left(\widetilde{x_{L}, x_{0}}\right)=\frac{1}{T_{L}}+\partial_{0}^{2} \omega_{1}+\partial_{0} \partial_{1} \omega_{1}-\frac{t_{1}}{T_{L}} \partial_{0} \partial_{1} \omega_{1}+\left(-1+t_{1} \partial_{0} \partial_{1} \omega_{1}\right) b\left(x_{L}, x_{0}\right) .
$$

For the other derivatives of $S\left(x_{L}, x_{0}\right)$, similar arguments hold, since we have

$$
\partial_{0} \partial_{L} S\left(\widetilde{x_{L}, x_{0}}\right)=\partial_{L} k_{1}^{*}\left(-1+t_{1} \partial_{0} \partial_{1} \omega_{1}\right)=\partial_{0} k_{L}^{*}\left(1-t_{L} \partial_{L} \partial_{L-1} \omega_{L}\right)
$$

and so on. Therefore we obtain (i').

(ii') To simplify the notation we put $l=j_{r-1}, m=j_{r}$ and $n=j_{r-1}$. We have 


$$
\begin{aligned}
& \left(\partial_{l}+\partial_{m}+\partial_{n}\right) \partial_{m}\left(\omega_{m, l+1}^{\#}\left(x_{m}, x_{l}\right)+\omega_{n, m+1}^{\#}\left(x_{n}, x_{m}\right)\right) \\
= & \left(\partial_{l}+\partial_{m}+\partial_{n}\right) \partial_{m}\left(S_{m, l+1}^{\#}\left(x_{m}, x_{l}\right)+S_{n, m+1}^{\#}\left(x_{n}, x_{m}\right)\right) \\
= & \partial_{m} k_{l+1}^{*}\left(t_{l+1} \partial_{l} \partial_{l+1} \omega_{l+1}-1\right)+\partial_{m} k_{m}^{*}\left(1-t_{m} \partial_{m} \partial_{m-1} \omega_{m}\right) \\
& +\partial_{m}^{2} \omega_{m}+\partial_{m} \partial_{m-1} \omega_{m}+\partial_{m}^{2} \omega_{m+1}+\partial_{m} \partial_{m+1} \omega_{m+1} \\
& +\partial_{m} k_{m+1}^{*}\left(t_{m+1} \partial_{m} \partial_{m+1} \omega_{m+1}-1\right)+\partial_{m} k_{n}^{*}\left(1-t_{n} \partial_{n} \partial_{n-1} \omega_{n}\right),
\end{aligned}
$$

where $k_{l+1}^{*}$ and $k_{m}^{*}$ are functions of $\left(x_{l}, x_{m}\right)$ and $k_{m+1}^{*}$ and $k_{n}^{*}$ are of $\left(x_{m}, x_{n}\right)$. We can show that

$$
\begin{aligned}
& \partial_{m} k_{l+1}^{*}\left(t_{l+1} \partial_{l} \partial_{l+1} \omega_{l+1}-1\right)+\partial_{m} k_{m}^{*}\left(1-t_{m} \partial_{m} \partial_{m-1} \omega_{m}\right) \\
& =\sum_{j=l+1}^{m-1} \phi_{j}\left(x_{j-1}^{*}, x_{j}^{*}, x_{j+1}^{*}\right) \partial_{m} x_{j}^{*}\left(x_{l}, x_{m}\right)
\end{aligned}
$$

where $\phi_{j}\left(x_{j-1}, x_{j}, x_{j+1}\right)=\left[\left(\partial_{j-1}+\partial_{j}+\partial_{j+1}\right) \partial_{j}\left(\omega_{j}+\omega_{j+1}\right)\right]\left(x_{j-1}, x_{j}, x_{j-1}\right)$. In fact we have

$$
\begin{aligned}
t_{l+1} \partial_{m} k_{l+1}^{*} & =\partial_{m} x_{l+1}^{*} . \\
t_{m} \partial_{m} k_{m}^{*} & =1-\partial_{m} x_{m-1}^{*}, \\
\partial_{m} k_{m}^{*}-\partial_{m} k_{l+1}^{*} & =(1, \ldots, 1) W\left(l+1, m ; X_{l, m}^{*}\right) \partial_{m} X_{l, m}^{*}+\partial_{m} \partial_{m-1} \omega_{m},
\end{aligned}
$$

where ${ }^{t} X_{l, m}^{*}=\left(x_{l+1}^{*}, \ldots, x_{m-1}^{*}\right)$ and $W\left(l+1, m ; X_{l, m}^{*}\right)$ is the Hessian matrix of $\sum_{j=l+1}^{m} \omega_{j}$ with respect to $\left(x_{l+1}, \ldots, x_{m-1}\right)$ at $X_{l, m}^{*}$ :

$$
\begin{aligned}
& W\left(l+1, m ; X_{l, m}^{*}\right) \\
& =\left(\begin{array}{cccc}
\partial_{l+1}^{2}\left(\omega_{l+1}+\omega_{l+2}\right) & \partial_{l+1} \partial_{l+2} \omega_{l+2} & 0 & \cdots \\
\partial_{l+1} \partial_{l+2} \omega_{l+2} & \partial_{l+2}^{2}\left(\omega_{l+2}+\omega_{l+3}\right) & \cdots & 0 \\
\vdots & \vdots & \ddots & \vdots \\
0 & \cdots & \cdots & \partial_{m-1}^{2}\left(\omega_{m-1}+\omega_{m}\right)
\end{array}\right) .
\end{aligned}
$$

Therefore we have

$$
\begin{aligned}
& \left(\partial_{l}+\partial_{m}+\partial_{n}\right) \partial_{m}\left(\omega_{m, l+1}^{\#}\left(x_{m}, x_{l}\right)+\omega_{n, m+1}^{\#}\left(x_{n}, x_{m}\right)\right) \\
& =\sum_{j=l+1}^{m-1} \phi_{j}\left(x_{j-1}^{*}, x_{j}^{*}, x_{j+1}^{*}\right) \partial_{m} x_{j}^{*}\left(x_{l}, x_{m}\right)+\phi_{m}\left(x_{m-1}^{*}, x_{m}^{*}, x_{m+1}^{*}\right) \\
& \quad+\sum_{j=m+1}^{n-1} \phi_{j}\left(x_{j-1}^{*}, x_{j}^{*}, x_{j+1}^{*}\right) \partial_{m} x_{j}^{*}\left(x_{m}, x_{n}\right) .
\end{aligned}
$$

When $\left(\bar{x}_{L}, \bar{x}_{j_{s}}, \ldots, \bar{x}_{j_{1}}, \bar{x}_{0}\right)$ is a solution of $(3.17),\left(\bar{x}_{L}, x_{L-1}^{*}\left(\bar{x}_{L}, \bar{x}_{j_{s}}\right), \ldots, x_{1}^{*}\left(\bar{x}_{j_{1}}\right.\right.$, $\left.\left.\bar{x}_{0}\right), \bar{x}_{0}\right)$ is a solution of (1.5). So summing the absolute value of (3.20) over $r$ (because $l=j_{r-1}, m=j_{r}$ and $\left.n=j_{r+1}\right)$ and substituting $\left(\bar{x}_{L}, \bar{x}_{j_{s}}, \ldots, \bar{x}_{j_{1}}, \bar{x}_{0}\right)$, we have 
(3.18) for $K=1$ by (3.12) and (H.1)(ii).

Next we show (3.18) for the case $K=2$. We can rewrite (3.20) as

$$
\begin{aligned}
& \left(\partial_{l}+\partial_{m}+\partial_{n}\right) \partial_{m}\left(\omega_{m, l+1}^{\#}\left(x_{m}, x_{l}\right)+\omega_{n, m+1}^{\#}\left(x_{n}, x_{m}\right)\right) \\
& =\sum_{j=l+1}^{n-1} \phi_{j}\left(x_{j}^{*}-t_{j} k_{j}^{*}, x_{j}^{*}, x_{j}^{*}+t_{j+1} k_{j+1}^{*}\right) p_{j}\left(x_{l}, x_{m}, x_{n}\right),
\end{aligned}
$$

where $p_{j}$ are bounded in $\mathscr{B}$ by $(3.12,13)$. Differentiating $(3.21)$ by $\left(\partial_{l}+\partial_{m}+\partial_{n}\right)$, we have

$$
\begin{aligned}
& \left(\partial_{l}+\partial_{m}+\partial_{n}\right)^{2} \partial_{m}\left(\omega_{m, l+1}^{\#}\left(x_{m}, x_{l}\right)+\omega_{n, m+1}^{\#}\left(x_{n}, x_{m}\right)\right) \\
& =\sum_{j=l+1}^{n-1}\left[\phi_{j}^{(2)}\left(\partial_{l}+\partial_{m}+\partial_{n}\right) x_{j}^{*} p_{j}-t_{j} \partial_{j-1} \phi_{j}\left(\partial_{l}+\partial_{m}+\partial_{n}\right) k_{j}^{*} p_{j}\right. \\
& \left.+t_{j+1} \partial_{j+1} \phi_{j}\left(\partial_{l}+\partial_{m}+\partial_{n}\right) k_{j+1}^{*} p_{j}+\phi_{j}\left(\partial_{l}+\partial_{m}+\partial_{n}\right) p_{j}\right],
\end{aligned}
$$

where $\phi_{j}^{(2)}=\left(\partial_{j-1}+\partial_{j}+\partial_{j+1}\right)^{2} \partial_{j}\left(\omega_{j}+\omega_{j+1}\right)$. On the other hand by $(3.10,11)$ we have

$$
\begin{aligned}
& \left(\partial_{l}+\partial_{m}+\partial_{n}\right) k_{j}^{*}=\left(\partial_{l}+\partial_{m}\right) k_{j}^{*}=q_{j}, \quad l+1 \leq j \leq m, \\
& \left(\partial_{l}+\partial_{m}+\partial_{n}\right) k_{j}^{*}=\left(\partial_{m}+\partial_{n}\right) k_{j}^{*}=q_{j}, \quad m+1 \leq j \leq n,
\end{aligned}
$$

where $q_{j}$ are bounded in $\mathscr{B}$. So from $(H .1)(i)$ and $(3.12,13)$ the right-hand side of (3.22) is of the form

$$
\sum_{j=l+1}^{n-1}\left[\phi_{j}^{(2)} p_{j}^{\prime}+\left(t_{j}+t_{j+1}\right) q_{j}^{\prime}+\phi_{j} p_{j}^{\prime \prime}\right]
$$

where $p_{j}^{\prime}, p_{j}^{\prime \prime}$ and $q_{j}^{\prime}$ are bounded in $\mathscr{B}$. Summing the absolute value of (3.23) over $r$ and substituting $\left(\bar{x}_{L}, \bar{x}_{j_{s}}, \ldots, \bar{x}_{j_{1}}, \bar{x}_{0}\right)$, by (H.1)(ii) we have (3.15) for $K=2$. For the other higher derivatives similar arguments hold. So (ii') is proved.

Next we consider the Hessian matrix at the critical point. The Hessian matrix of $S$ is equal to $H(L)+W(1, L ; x)$, where

$$
H(L)=\left(\begin{array}{cccccc}
\frac{1}{t_{1}}+\frac{1}{t_{2}} & -\frac{1}{t_{2}} & 0 & \cdots & \cdots & \\
-\frac{1}{t_{2}} & \frac{1}{t_{2}}+\frac{1}{t_{3}} & -\frac{1}{t_{3}} & 0 & \cdots & \\
0 & -\frac{1}{t_{3}} & \frac{1}{t_{3}}+\frac{1}{t_{4}} & \cdots & \cdots & \\
\vdots & \vdots & \vdots & \ddots & \cdots & \\
0 & \cdots & \cdots & \cdots & -\frac{1}{t_{L-1}} & \frac{1}{t_{L-1}}+\frac{1}{t_{L}}
\end{array}\right)
$$


and for $x=\left(x_{1}, \ldots, x_{L-1}\right)$,

$$
W(1, L ; x)=\left(\begin{array}{ccccc}
\partial_{1}^{2}\left(\omega_{1}+\omega_{2}\right) & \partial_{1} \partial_{2} \omega_{2} & 0 & \cdots & \\
\partial_{1} \partial_{2} \omega_{2} & \partial_{2}^{2}\left(\omega_{2}+\omega_{3}\right) & \partial_{2} \partial_{3} \omega_{3} & 0 & \\
\vdots & \vdots & \vdots & \ddots & \partial_{L-1} \partial_{L-2} \omega_{L-1} \\
0 & \cdots & \cdots & \cdots & \partial_{L-1}^{2}\left(\omega_{L-1}+\omega_{L}\right)
\end{array}\right) .
$$

We have

$$
\operatorname{det} H(L)=\frac{T_{L}}{t_{1} \cdots t_{L}} .
$$

Let $G(L)$ be the inverse of $H(L)$. Then its (ij) entry is

$$
\begin{aligned}
(G(L))_{1 \jmath} & =\frac{T_{i}\left(T_{L}-T_{j}\right)}{T_{L}}, \quad \text { if } 1 \leq i \leq j \leq L-1, \\
& =\frac{T_{j}\left(T_{L}-T_{i}\right)}{T_{L}}, \quad \text { if } 1 \leq j \leq i \leq L-1 .
\end{aligned}
$$

We set

$$
G_{1}(L)=\frac{1}{T_{L}}\left(\begin{array}{ccccc}
t_{1} & t_{1} & t_{1} & \cdots & t_{1} \\
-\left(t_{3}+\cdots+t_{L}\right) & T_{2} & T_{2} & \cdots & T_{2} \\
-\left(t_{4}+\cdots+t_{L}\right) & -\left(t_{4}+\cdots+t_{L}\right) & T_{3} & \cdots & T_{3} \\
\vdots & \vdots & \vdots & \vdots & \vdots \\
-t_{L} & -t_{L} & \cdots & -t_{L} & T_{L-1}
\end{array}\right)
$$

and

$$
G_{2}(L)=\left(\begin{array}{cccc}
t_{3} & 0 & \cdots & 0 \\
t_{3} & t_{3} & 0 & \cdots \\
\vdots & \vdots & \vdots & \vdots \\
t_{L} & t_{L} & \cdots & t_{L}
\end{array}\right)
$$

Then we have $G(L)=G_{1}(L) G_{2}(L)$.

We use two norms $\|x\|_{\infty}=\max _{1 \leq j \leq L-1}\left|x_{j}\right|$ and $\|x\|_{1}=\sum_{j=1}^{L-1}\left|x_{j}\right|$ for any $x \in \mathbf{R}^{L-1}$. 
Lemma 3.8. Let $x^{*}=\left(x_{1}^{*}, \ldots, x_{L-1}^{*}\right)=\left(x_{1}^{*}\left(x_{0}, x_{L}\right), \ldots, x_{L-1}^{*}\left(x_{0}, x_{L}\right)\right)$ be the critical point. Then we have for any $u \in \mathbf{R}^{L-1}$,

$$
\begin{gathered}
\left\|G_{1}(L) u\right\|_{\infty} \leq\|u\|_{1}, \\
\left\|G_{2}(L) W\left(1, L ; x^{*}\right) u\right\|_{1} \leq\left(9 \kappa_{2}+B_{1}\right) T_{L}\|u\|_{\infty} \text { and } \\
\left\|G(L) W\left(1, L ; x^{*}\right) u\right\|_{\infty} \leq\left(9 \kappa_{2}+B_{1}\right) T_{L}\|u\|_{\infty} .
\end{gathered}
$$

Proof. For the proof we have only to sum the magnitudes of all component of the matrix $G_{2}(L) W\left(1, L ; x^{*}\right)$. Since the first column of $G_{2}(L) W\left(1, L ; x^{*}\right)$ is

$$
h_{1}=\left(\begin{array}{c}
t_{2} \partial_{1}^{2}\left(\omega_{1}+\omega_{2}\right) \\
t_{3}\left(\partial_{1}^{2}\left(\omega_{1}+\omega_{2}\right)+\partial_{1} \partial_{2} \omega_{2}\right) \\
t_{4}\left(\partial_{1}^{2}\left(\omega_{1}+\omega_{2}\right)+\partial_{1} \partial_{2} \omega_{2}\right) \\
\cdots \\
t_{L}\left(\partial_{1}^{2}\left(\omega_{1}+\omega_{2}\right)+\partial_{1} \partial_{2} \omega_{2}\right)
\end{array}\right),
$$

we have $\left\|h_{1}\right\|_{1} \leq 3 \kappa_{2} T_{L}$. For $2 \leq j \leq L-1$, the $j$-th column of $G_{2}(L) W(1, L$; $\left.x^{*}\right)$ is

$$
h_{i}=\left(\begin{array}{c}
0 \\
\vdots \\
0 \\
t_{j} \partial_{j-1} \partial_{j} \omega_{j} \\
t_{j+1}\left(\partial_{j-1} \partial_{j} \omega_{j}+\partial_{j}^{2}\left(\omega_{j}+\omega_{j+1}\right)\right) \\
t_{j+2} \phi_{j} \\
\vdots \\
t_{L} \phi_{j}
\end{array}\right),
$$

where $\phi_{j}=\left(\partial_{j-1}+\partial_{j}+\partial_{j+1}\right) \partial_{j}\left(\omega_{j}+\omega_{j+1}\right)$. So we have $\left\|h_{j}\right\|_{1} \leq 3 \kappa_{2}\left(t_{j}+\right.$ $\left.t_{j+1}\right)+T_{L}\left|\phi_{j}\right|$. Therefore by (H.1)(ii), we have $\sum_{j=1}^{L-1}\left\|h_{j}\right\|_{1} \leq\left(9 \kappa_{2}+B_{1}\right) T_{L}$.

Let $x_{1}^{*}$ be the critical point of $S_{2}\left(x_{2}, x_{1}\right)+S_{1}\left(x_{1}, x_{0}\right)$ with respect to $x_{1}$. We define a function $D\left(S_{2}+S_{1} ; x_{2}, x_{0}\right)$ through the Hessian determinant at $x_{1}^{*}$ in the following way:

$$
\operatorname{det} \operatorname{Hess}_{x_{1}^{*}}\left(S_{2}+S_{1}\right)=\frac{t_{1}+t_{2}}{t_{1} t_{2}} D\left(S_{2}+S_{1} ; x_{2}, x_{0}\right) .
$$

For $m>k+1$ we define $D\left(x_{m}, x_{k}\right)$ by 


$$
\operatorname{det}\left(\operatorname{Hess}_{\left(x_{m-1}^{*}, \cdots, x_{k+1}^{*}\right)}\left(S_{m}+\cdots+S_{k+1}\right)\right)=\frac{t_{k+1}+\cdots+t_{m}}{t_{k+1} \cdots t_{m}} D\left(x_{m}, x_{k}\right) .
$$

Lemma 3.9. Let $0<T^{1}<T$ with $2\left(9 \kappa_{2}+B_{1}\right) T^{1}<1$. If $T_{L}<T^{1}$, then we have

$$
D\left(x_{L}, x_{0}\right)=\left.\prod_{k=2}^{L} D\left(S_{k}+S_{k-1,1}^{\#} ; x_{k}, x_{0}\right)\right|_{\left(x_{L-1}, \cdots, x_{1}\right)=\left(x_{L-1}^{*}, \cdots, x_{1}^{*}\right)} .
$$

Proof. When $2\left(9 \kappa_{2}+B_{1}\right) T_{L}<1$, we have that

$$
\begin{aligned}
& \operatorname{det} \operatorname{Hess}_{\left(x_{k-1}^{*}, \cdots, x_{1}^{*}\right)}\left(S_{k}\left(x_{k}, x_{k-1}^{*}\right)+\cdots+S_{1}\left(x_{1}^{*}, x_{0}\right)\right) \\
& =\frac{t_{k}+\cdots+t_{1}}{t_{k} \cdots t_{1}} \operatorname{det}\left(I+G(k) W\left(1, k ; x^{*}\right)\right) \neq 0, \quad k=2, \ldots, L,
\end{aligned}
$$

because $I+G(k) W\left(1, k ; x^{*}\right)$ has the inverse matrix by Lemma 3.8. So applying [5, Proposition 2.6], we can prove the lemma by induction on $L$, similarly to [5, Proposition 2.8].

Lemma 3.10. If $T_{L}<T^{1}$, we can write

$$
D\left(x_{L}, x_{0}\right)=1+T_{L} g\left(x_{L}, x_{0}\right),
$$

where $g\left(x_{L}, x_{0}\right)$ remains bounded in $\mathscr{B}(\mathbf{R} \times \mathbf{R})$ uniformly with respect to $t_{1}, \ldots, t_{L}$.

Proof. By Lemmas 3.6, 3.7 and 3.9, we can write

$$
\begin{aligned}
D\left(x_{L}, x_{0}\right) & =\left.\prod_{k=2}^{L} D\left(S_{k}+S_{k-1,1}^{*} ; x_{k}, x_{0}\right)\right|_{\left(x_{L-1}, \cdots, x_{1}\right)=\left(x_{L-1}^{*}, \cdots, x_{1}^{*}\right)} \\
& =\left.\prod_{k=2}^{L}\left(1+\frac{t_{k} T_{k-1}}{T_{k}} \partial_{k-1}^{2}\left(\omega_{k}+\omega_{k-1,1}^{*}\right)\right)\right|_{\left(x_{L-1}, \cdots, x_{1}\right)=\left(x_{L-1}^{*}, \cdots, x_{1}^{*}\right)} \\
& =\prod_{k=2}^{L}\left(1+t_{k} p_{k}\left(x_{L}, x_{0}\right)\right),
\end{aligned}
$$

where $p_{k}\left(x_{L}, x_{0}\right)$ are bounded in $\mathscr{B}(\mathbf{R} \times \mathbf{R})$. So the lemma is proved.

It is noted that Lemma 3.10 differs from Fujiwara [5, Proposition 2.10] in the power of $T_{L}$; our power is 1 while his is 2 . 


\section{Key lemma}

In this section we write down key lemmas to prove Theorems 1 and 2. Their assertions are the same as those of [5] except for the form of the phase function. Let $S_{j}\left(t_{j}, x, y\right)=\frac{|x-y|^{2}}{2 t_{j}}+\omega_{j}\left(t_{j}, x, y\right), i=1,2$ be phase functions satisfying (H.1)(i), and $a(x, z, y)$ an amplitude function in $\mathscr{B}(\mathbf{R} \times \mathbf{R} \times \mathbf{R})$. We set $\tau=$ $t_{1} t_{2} /\left(t_{1}+t_{2}\right)$ and $E=\nu i /(2 \pi)$. The notation $D\left(S_{2}+S_{1} ; x, y\right)$ is given in $\S 3$.

Lemma 4.1. Assume that $8 \tau \kappa_{2} \leq 1$. Then

$$
\begin{aligned}
& \left(\frac{E}{t_{1}}\right)^{1 / 2}\left(\frac{E}{t_{2}}\right)^{1 / 2} \int_{\mathbf{R}} e^{-i \nu\left(S_{1}\left(t_{1}, x, z\right)+S_{2}\left(t_{2}, z, y\right)\right)} a(x, z, y) d z \\
& =\left(\frac{E}{t_{1}+t_{2}}\right)^{1 / 2} e^{-i \nu S_{2,1}^{*}(x, y)} D\left(S_{2}+S_{1} ; x, y\right)^{-1 / 2} b(x, y),
\end{aligned}
$$

with

$$
\begin{aligned}
& b(x, y)=a\left(x, z^{*}, y\right)+\left(\frac{\tau}{i \nu}\right) D\left(S_{2}+S_{1} ; x, y\right)^{-1}\left[\frac{1}{2}\left(\Delta_{z} a\right)\left(x, z^{*}, y\right)\right. \\
& \left.+\tau D\left(S_{2}+S_{1} ; x, y\right)^{-1} r_{1}(x, y)\right]+\left(\frac{\tau}{i \nu}\right)^{2} D\left(S_{2}+S_{1} ; x, y\right)^{-2} r_{2}(x, y),
\end{aligned}
$$

where $\Delta_{z}$ is the Laplacian with respect to $z$. For any $m \geq 0$ there exist $C_{m}$ and $M(m)$ such that if $|\alpha|,|\beta| \leq m$,

$$
\left|\partial_{x}^{\alpha} \partial_{y}^{\beta} r_{1}(x, y)\right|+\left|\partial_{x}^{\alpha} \partial_{y}^{\beta} r_{2}(x, y)\right| \leq C_{m} \max \sup _{z}\left|\partial_{x}^{\alpha^{\prime}} \partial_{y}^{\beta^{\prime}} \partial_{z}^{\gamma^{\prime}} a(x, z, y)\right|,
$$

where $\max$ is taken for $\alpha^{\prime} \leq \alpha, \beta^{\prime} \leq \beta$ and $\gamma^{\prime} \leq M(m)$. $M(m)$ can be chosen as $2 m+4 d+2$.

Proof. We have only to apply the stationary phase method (cf. [1, Theorem 4.1].)

The next lemma plays an important role.

Lemma 4.2. For the phase function we assume (H.1). Let $a\left(x_{L}, \ldots, x_{0}\right)$ be an amplitude function in $\mathscr{B}\left(\mathbf{R}^{d(L+1)}\right)$. Then there exists a constant $\delta>0$ such that if $T_{L}<\delta$ then

$$
I\left(\left\{t_{j}\right\}, S, a, \nu\right)\left(x_{L}, x_{0}\right)=\left(\frac{\nu i}{2 \pi T_{L}}\right)^{1 / 2} \exp \left(-i \nu S\left(x_{L}, x_{0}\right)\right) b\left(x_{L}, x_{0}\right) .
$$


For any $m \geq 0$ there exist constants $C_{m}$ and $K(m)$ such that if $\left|\alpha_{0}\right|,\left|\alpha_{L}\right| \leq m$,

$$
\left|\partial_{L}^{\alpha_{L}} \partial_{0}^{\alpha_{0}} b\left(x_{L}, x_{0}\right)\right| \leq C_{m}^{L} \max _{\beta} \sup _{x_{L-1}, \cdots, x_{1}}\left|\partial_{L}^{\beta_{L}} \partial_{L-1}^{\beta_{L-1}} \cdots \partial_{0}^{\beta_{0}} a\left(x_{L}, \ldots, x_{0}\right)\right|,
$$

where $\max$ is taken for $\left(\beta_{L}, \ldots, \beta_{0}\right)$ satisfying $\beta_{0} \leq \alpha_{0}, \beta_{L} \leq \alpha_{L}$ and $\left|\beta_{j}\right| \leq K(m)$, $j=1, \ldots, L-1 . C_{m}$ and $K(m)$ do not depend on $L, \nu$ and $a$. We can choose $K(m)=12 m+48 d+21$.

For the proof of this lemma we refer to $\S 3$ of Fujiwara [5]. Though the assumption here for the phase function is more general than that of [5], the arguments there apply to our case word by word.

\section{Proof of Theorems 1 and 2}

The arguments in the proof of Theorems 1 and 2 will be the same as those in [5] except for taking (1.8b) in (H.2) into consideration.

For any $l>k$ we put $T(l, k)=t_{l}+\cdots+t_{k}$ and $T(k, k)=t_{k}$. We set $E=\nu i /(2 \pi)$. Let $\delta$ be as in Lemma 4.2 and let $T^{1}$ be as in Lemma 3.9. Put $\delta^{\prime}=\min \left(\delta, T^{1}\right)$. When $T_{L}<\delta^{\prime}$, we consider the oscillatory integral

$$
\begin{aligned}
& I\left(\left\{t_{j}\right\}, S, \alpha, \nu\right)= \\
& \prod_{j=1}^{L}\left(\frac{E}{t_{j}}\right)^{1 / 2} \int_{\mathbf{R}^{(L-1)}} \exp \left(-i \nu \sum_{j=1}^{L} S_{j}\left(x_{j}, x_{j-1}\right)\right) a\left(x_{L}, \ldots, x_{0}\right) \prod_{j=1}^{L-1} d x_{j} .
\end{aligned}
$$

First we perform integration over $x_{1}$ space. Applying the stationary phase method, we have

$$
\begin{aligned}
& \left(\frac{E}{t_{2}}\right)^{1 / 2}\left(\frac{E}{t_{1}}\right)^{1 / 2} \int_{\mathbf{R}} e^{-i \nu\left(S_{2}\left(x_{2}, x_{1}\right)+S_{1}\left(x_{1}, x_{0}\right)\right)} a\left(x_{L}, \ldots, x_{0}\right) d x_{1} \\
& =\left(\frac{E}{T(2,1)}\right)^{1 / 2} e^{-i \nu S_{2,1}^{\#}\left(x_{2}, x_{0}\right)}\left(\left(S_{1} a\right)\left(x_{L}, \ldots, x_{2}, x_{0}\right)+\left(R_{1} a\right)\left(x_{L}, \ldots, x_{2}, x_{0}\right)\right),
\end{aligned}
$$

where $S_{1} a$ is the main term

$$
\left(S_{1} a\right)\left(x_{L}, \ldots, x_{2}, x_{0}\right)=a\left(x_{L}, \ldots, x_{2}, x_{0}\right) D\left(S_{2}+S_{1} ; x_{2}, x_{0}\right)^{-1 / 2},
$$

and $R_{1} a$ is the remainder term.

Next, we integrate $S_{1} a$ over $x_{2}$ space and apply the stationary phase method, then we have 


$$
\begin{aligned}
& \left(\frac{E}{t_{3}}\right)^{1 / 2}\left(\frac{E}{T(2,1)}\right)^{1 / 2} \int_{\mathbf{R}} e^{-i \nu\left(S_{3}\left(x_{3}, x_{2}\right)+S_{2,1}^{*}\left(x_{2}, x_{0}\right)\right)}\left(S_{1} a\right)\left(x_{L}, \ldots, x_{2}, x_{0}\right) d x_{2} \\
& =\left(\frac{E}{T(3,1)}\right)^{1 / 2} e^{-i \nu S_{3,1}^{*}\left(x_{3}, x_{0}\right)}\left(\left(S_{2} S_{1} a\right)\left(x_{L}, \ldots, x_{3}, x_{0}\right)+\left(R_{2} S_{1} a\right)\left(x_{L}, \ldots, x_{3}, x_{0}\right)\right) .
\end{aligned}
$$

Here $S_{2} S_{1} a$ is the main term and $R_{2} S_{1} a$ is the remainder term, i.e.

$$
\left(S_{2} S_{1} a\right)\left(x_{L}, \ldots, x_{3}, x_{0}\right)=\left(S_{1} a\right)\left(x_{L}, \ldots, x_{3}, x_{2}^{*}, x_{0}\right) D\left(S_{3}+S_{2,1}^{\#} ; x_{3}, x_{0}\right)^{-1 / 2},
$$

where $x_{2}^{*}$ is the critical point of $S_{3}+S_{2,1}^{\#}$ with respect to $x_{2}$.

Repeating this process $L-1$ times, by Lemma 3.9 we have the main term of Theorems 1 and 2:

$$
\begin{aligned}
& \left(\frac{E}{T_{L}}\right)^{1 / 2} e^{-i \nu S E_{1}, 1\left(x_{L}, x_{0}\right)}\left(S_{L-1} S_{L-2} \cdots S_{1} a\right)\left(x_{L}, x_{0}\right) \\
& =\left(\frac{E}{T_{L}}\right)^{1 / 2} e^{-i \nu S_{t, 1}^{\#}\left(x_{L}, x_{0}\right)} D\left(x_{L}, x_{0}\right)^{-1 / 2} a\left(x_{L}, x_{0}\right) .
\end{aligned}
$$

Next we treat the remainder term. Since $\left(R_{1} a\right)\left(x_{L}, \ldots, x_{2}, x_{0}\right)$ has complicated structure as a function of $x_{2}$, we postpone integration over $x_{2}$ space of the term including $\left(R_{1} a\right)\left(x_{L}, \ldots, x_{2}, x_{0}\right)$ and perform integration over $x_{3}$ space beforehand. The stationary phase method gives

$$
\begin{aligned}
& \left(\frac{E}{t_{4}}\right)^{1 / 2}\left(\frac{E}{t_{3}}\right)^{1 / 2}\left(\frac{E}{T(2,1)}\right)^{1 / 2} \int_{\mathbf{R}} e^{-\imath \nu\left(S_{4}\left(x_{4}, x_{3}\right)+S_{3}\left(x_{3}, x_{2}\right)+S_{2,1}^{\#}\left(x_{2}, x_{0}\right)\right)} \\
& \quad \quad \times\left(R_{1} a\right)\left(x_{L}, \ldots, x_{2}, x_{0}\right) d x_{3} \\
& =\left(\frac{E}{T(4,3)}\right)^{1 / 2}\left(\frac{E}{T(2,1)}\right)^{1 / 2} e^{-i \nu\left(S_{4,3}^{*}\left(x_{4}, x_{2}\right)+S_{2,1}^{\#}\left(x_{2}, x_{0}\right)\right)} \\
& \quad \times\left(\left(S_{3} R_{1} a\right)\left(x_{L}, \ldots, x_{4}, x_{2}, x_{0}\right)+\left(R_{3} R_{1} a\right)\left(x_{L}, \ldots, x_{4}, x_{2}, x_{0}\right)\right),
\end{aligned}
$$

where $S_{3} R_{1} a$ is the main term and $R_{3} R_{1} a$ is the remainder i.e.

$$
\left(S_{3} R_{1} a\right)\left(x_{L}, \ldots, x_{4}, x_{2}, x_{0}\right)=\left(R_{1} a\right)\left(x_{L}, \ldots, x_{4}, x_{2}, x_{0}\right) D\left(S_{4}+S_{3} ; x_{4}, x_{2}\right)^{-1 / 2}
$$

Similarly, we skip integration over $x_{3}$ space of the term including $\left(R_{2} S_{1} a\right)\left(x_{L}, \ldots\right.$, $x_{3}, x_{0}$ ) and integrate it over $x_{4}$ space.

We continue this process: if $R_{k}$ appears we skip integration over $x_{k+1}$ space. Thus we can write $I\left(\left\{t_{j}\right\}, S, a, \nu\right)$ as

$$
I\left(\left\{t_{j}\right\}, S, a, \nu\right)\left(x_{L}, x_{0}\right)=A_{0}\left(x_{L}, x_{0}\right)+\sum^{\prime} A_{j_{S} j_{s-1} \cdots j_{1}}\left(x_{L}, x_{0}\right) .
$$

Here the main term is 


$$
A_{0}\left(x_{L}, x_{0}\right)=\left(\frac{E}{T_{L}}\right)^{1 / 2} e^{-i \nu S_{L, 1}^{\#}\left(x_{L}, x_{0}\right)} D\left(x_{L}, x_{0}\right)^{-1 / 2} a\left(x_{L}, x_{0}\right) .
$$

The sum $\Sigma^{\prime}$ is taken over the sequences of integers $\left(j_{s}, j_{s-1}, \ldots, j_{1}\right)$ with the property

$$
0=j_{0}<j_{1}-1<j_{1}<j_{2}-1<\cdots<j_{s}-1<j_{s} \leq L=j_{s+1} .
$$

The summand is

$$
\begin{aligned}
& A_{j_{s} j_{s-1} \ldots j_{1}}\left(x_{L}, x_{0}\right) \\
= & \prod_{u=1}^{s+1}\left(\frac{E}{T\left(j_{u}, j_{u-1}+1\right)}\right)^{1 / 2} \int_{\mathbf{R}^{s}} \exp \left(-i \nu S_{j_{s} j_{s-1} \ldots j_{1}}^{\#}\left(x_{L}, x_{j_{s}}, \ldots, x_{j_{1}}, x_{0}\right)\right) \\
& \times b_{j_{s} j_{s-1} \ldots j_{1}}\left(x_{L}, x_{j_{s}}, \ldots, x_{j_{1}}, x_{0}\right) \prod_{u=1}^{s} d x_{j_{u}} .
\end{aligned}
$$

The amplitude of this is

$$
b_{j_{s} j_{s-1} \ldots j_{1}}\left(x_{L}, x_{j_{s}}, \ldots, x_{j_{1}}, x_{0}\right)=\left(Q_{L-1} Q_{L-2} \cdots Q_{1} a\right)\left(x_{L}, x_{j_{s}}, \ldots, x_{j_{1}}, x_{0}\right),
$$

where

$$
\begin{aligned}
Q_{j} & =\mathrm{Id}, \quad \text { if } j=j_{s}, j_{s-1}, \ldots, j_{1}, \\
& =\mathrm{R}_{j}, \quad \text { if } j=j_{s}-1, j_{s-1}-1, \ldots, j_{1}-1 \\
& =S_{j}, \quad \text { otherwise. }
\end{aligned}
$$

The phase is

$$
S_{j_{s} j_{s-1} \ldots j_{1}}^{\#}\left(x_{L}, x_{j_{s}}, \ldots, x_{j_{1}}, x_{0}\right)=\sum_{u=1}^{s+1} S_{j_{u} j_{u-1}+1}^{\#}\left(x_{j_{u}}, x_{j_{u-1}}\right),
$$

where we understand $S_{j_{s+1}, j_{s}+1}^{\#}=0$ when $j_{s}=L$, and $S_{j_{s+1}, j_{s}+1}^{\#}=S_{L, L}^{\#}=S_{L}\left(x_{L}\right.$, $x_{L-1}$ ) when $j_{s}=L-1$. In (5.11), when $j_{s}=L$ then the integration over $x_{j_{s}}$ is not performed. Moreover we understand $\frac{E}{T\left(j_{s+1}, j_{s}+1\right)}=1$ when $j_{s}=L$, and $T\left(j_{s+1}\right.$, $\left.j_{s}+1\right)=T(L, L)=t_{L}$ when $j_{s}=L-1$.

Note. Fujiwara [5] did not take the case $j_{s}=L$ into consideration in the sum of (5.9).

By Lemma 3.7 we know that (5.13) satisfies (H.1). So we can apply Lemma 4.2 to $A_{j_{s} j_{s-1} \ldots j_{1}}$ and obtain

$$
A_{j_{s} j_{s-1} \cdots j_{1}}\left(x_{L}, x_{0}\right)=\left(\frac{E}{T_{L}}\right)^{1 / 2} e^{-i \nu S_{L, 1}^{\#}\left(x_{L}, x_{0}\right)} a_{j_{s} j_{s-1} \cdots j_{1}}\left(x_{L}, x_{0}\right)
$$


where $a_{s_{s} j_{s-1} \ldots j_{1}}$ satisfies the estimate: For any $m \geq 0$ there exist $C_{m}$ and $K(m)$ such that if $\left|\alpha_{L}\right|,\left|a_{0}\right| \leq m$,

(a) when $j_{s}<L$,

(5.14a) $\left|\partial_{L}^{\alpha_{L}} \partial_{0}^{\alpha_{0}} a_{j_{s} j_{s-1} \ldots j_{1}}\left(x_{L}, x_{0}\right)\right|$

$$
\leq C_{m}^{s} \max \sup _{x_{j u}, u=1, \cdots, s}\left|\partial_{L}^{\beta_{L}} \partial_{j_{s}}^{\beta_{j_{s}}} \ldots \partial_{j_{1}}^{\beta_{j_{1}}} \partial_{0}^{\beta_{0}} b_{j_{s} j_{s-1} \ldots j_{1}}\left(x_{L}, x_{j_{s}}, \ldots, x_{j_{1}}, x_{0}\right)\right|
$$

where $\max$ is taken for $\beta_{L} \leq a_{L}, \beta_{0} \leq \alpha_{0}$ and $\beta_{j_{u}} \leq K(m)=12 m+48+21$, $u=1, \ldots, s$,

(b) when $j_{s}=L$,

$$
\begin{aligned}
& \left|\partial_{L}^{\alpha_{L}} \partial_{0}^{\alpha_{0}} a_{j_{s} j_{s-1} \ldots j_{1}}\left(x_{L}, x_{0}\right)\right| \\
& \leq C_{m}^{s} \max \sup _{x_{j_{u}}, u=1, \cdots, s-1}\left|\partial_{L}^{\beta_{L}} \partial_{j_{s-1}}^{\beta_{s_{s-1}}} \cdots \partial_{j_{1}}^{\beta_{j_{1}}} \partial_{0}^{\beta_{0}} b_{j_{s} j_{s-1} \cdots j_{1}}\left(x_{L}, x_{j_{s-1}}, \ldots, x_{j_{1}}, x_{0}\right)\right|
\end{aligned}
$$

where $\max$ is taken for $\beta_{L} \leq \alpha_{L}, \beta_{0} \leq \alpha_{0}$ and $\beta_{j_{u}} \leq K(m)=12 m+48+21$, $u=1, \ldots, s-1$. So we have

$$
\begin{gathered}
I\left(\left\{t_{j}\right\}, S, a, \nu\right)=\left(\frac{E}{T_{L}}\right)^{1 / 2} e^{-i \nu S_{L, 1}^{*}\left(x_{L}, x_{0}\right)} D\left(x_{L}, x_{0}\right)^{-1 / 2}\left(a\left(x_{L}, x_{0}\right)+r\left(x_{L}, x_{0}\right)\right), \\
r\left(x_{L}, x_{0}\right)=D\left(x_{L}, x_{0}\right)^{1 / 2} \sum^{\prime} a_{j_{S} j_{s-1} \ldots j_{1}}\left(x_{L}, x_{0}\right)
\end{gathered}
$$

Therefore from $(5.14 \mathrm{a}, \mathrm{b}, 15)$ we see that we have only to estimate $b_{j_{s} j_{s-1} \ldots j_{1}}$ to prove Theorems 1 and 2 .

Proof of Theorem 1. Assume (H.2).

Lemma 5.1. Let $T_{L}<\delta^{\prime}$. Then for any $m \geq 0$ there exist constants $C_{m, 1}$ and $M(m)$ such that for any $\alpha_{0}, \alpha_{L}, \alpha_{j_{u}} \leq m, 1 \leq u \leq s$,

$$
\begin{aligned}
& \left|\partial_{L}^{\alpha_{L}} \partial_{0}^{\alpha_{0}} \prod_{u=1}^{s} \partial_{j_{u}}^{\alpha_{j_{u}}} b_{j_{s} j_{s-1} \ldots j_{1}}\left(x_{L}, x_{j_{s}}, \ldots, x_{j_{1}}, x_{0}\right)\right| \leq C_{m, 1}^{s}\left(\prod_{u=1}^{s} \nu^{-1} t_{j_{u}}\right) \\
& \quad \times \max \sup \left|\partial_{L}^{\beta_{L}} \partial_{0}^{\beta_{0}} \prod_{u=1}^{s} \partial_{j_{u}-1}^{\beta_{j_{u}-1}} \partial_{j_{u}}^{\beta_{j_{u}}} a\left(\widetilde{x_{L}, x_{j_{s}}}, x_{j_{s}-1}, x_{j_{s-1}}, \ldots, x_{j_{1}-1}, x_{0}\right)\right|
\end{aligned}
$$

where max is taken for $\beta_{L} \leq \alpha_{L}, \beta_{0} \leq \alpha_{0}, \beta_{j_{u}} \leq \alpha_{j_{u}}$ and $\beta_{j_{u}-1} \leq M(m)$ and sup is taken for $x_{j_{u}-1}, 1 \leq u \leq s$. Here when $j_{s}=L$, the notation $\partial_{L}^{\alpha_{L}}$ appears only once and we understand $x_{L}, x_{j_{s}}=x_{j_{s}}$ on both the sides of the inequality (5.16). We can choose $M(m)=2 m+4+2$.

We assume Lemma 5.1 for the moment and prove Theorem 1. From (H.2) the 
right-hand side of (5.16) is majorized by $C_{m, 1}^{s}\left(\Pi_{u=1}^{s} \nu^{-1} t_{j_{u}}\right) A_{M(m)} X_{M(m)}^{s}$. So combining $\left(5.14 \mathrm{a}\right.$, b) with Lemma 5.1 , we have with $m^{\prime}=K(m)$

$$
\left|\partial_{L}^{\alpha_{L}} \partial_{0}^{\alpha_{0}} a_{j_{s} j_{s-1} \cdots j_{1}}\left(x_{L}, x_{0}\right)\right| \leq C_{m}^{s} C_{m^{\prime}, 1}^{s}\left(\prod_{u=1}^{s} \nu^{-1} t_{j_{u}}\right) A_{M\left(m^{\prime}\right)} X_{M\left(m^{\prime}\right)}^{s} .
$$

It follows with (5.15) that

$$
\begin{aligned}
\left|\partial_{L}^{\alpha_{L}} \partial_{0}^{\alpha_{0}} r\left(x_{L}, x_{0}\right)\right| & \leq\left|\left(\sum^{\prime} C_{m}^{s} C_{m^{\prime}, 1}^{s} X_{M\left(m^{\prime}\right)}^{s} \prod_{u=1}^{s}\left(\nu^{-1} t_{j_{u}}\right)\right)\right| A_{M\left(m^{\prime}\right)} \\
& \leq\left(\prod_{j=1}^{L}\left(1+C_{m} C_{m^{\prime}, 1} X_{M\left(m^{\prime}\right)} \nu^{-1} t_{j_{u}}\right)-1\right) A_{M\left(m^{\prime}\right)}
\end{aligned}
$$

This is the estimate $(1.10)$ in Theorem 1 with $M\left(m^{\prime}\right)=M(K(m))=2(12 m+48$ $+21)+4+2$.

Lemma 5.1 follows immediately from the next lemma. For any sequence of integers $0<k_{1}-1<k_{1}<k_{2}-1<\cdots<k_{r}-1<k_{r} \leq L$, we set

$$
\begin{aligned}
& p_{k_{r} k_{r-1}} \cdots k_{1} \\
& \quad=\left(x_{L}, x_{L-1}, \ldots, x_{k_{r}+1}, x_{k_{r}}, x_{k_{r-1}}, \ldots, x_{k_{1}}, x_{0}\right) \\
& =\left(Q_{k_{r}} Q_{k_{r}-1} \cdots Q_{1} a\right)\left(x_{L}, x_{L-1}, \ldots, x_{k_{r}+1}, x_{k_{r}}, x_{k_{r-1}}, \ldots, x_{k_{1}}, x_{0}\right),
\end{aligned}
$$

where

$$
\begin{aligned}
Q_{j} & =\mathrm{Id}, \quad \text { if } j=k_{r}, k_{r-1}, \ldots, k_{1}, \\
& =R_{j}, \quad \text { if } j=k_{r}-1, k_{r-1}-1, \ldots, k_{1}-1, \\
& =S_{j}, \quad \text { otherwise. }
\end{aligned}
$$

Lemma 5.2. For any $m \geq 0$ there exist constants $C_{m, 2}$ and $M(m)$ such that for arbitrary $\alpha_{L}$, if $\alpha_{0}, \alpha_{k_{j}} \leq m, 1 \leq j \leq r$, then

$$
\begin{aligned}
& \left|\partial_{L}^{\alpha_{L}} \partial_{0}^{\alpha_{0}} \prod_{j=1}^{r} \partial_{k_{j}}^{\alpha_{k_{j}}} p_{k_{r} k_{r-1} \ldots k_{1}}\left(\bar{x}_{L}, x_{k_{r}}, x_{k_{r-1}}, \ldots, x_{k_{1}}, x_{0}\right)\right| \\
& \leq C_{m, 2}^{r} \prod_{j=1}^{r}\left(\frac{t_{k_{j}} T\left(k_{j}-1, k_{j-1}+1\right)}{\nu T\left(k_{j}, k_{j-1}+1\right)}\right) \\
& \times \max \sup \left|\partial_{L}^{\alpha_{L}} \partial_{0}^{\beta_{0}} \prod_{j=1}^{r} \partial_{k_{j}-1}^{\beta_{k j-1}} \partial_{k_{j}}^{\beta_{k_{j}}} a\left(\widetilde{x_{L},} x_{k_{r}}, \widetilde{x_{k_{r}-1}}, x_{k_{r-1}}, \ldots, \widetilde{x_{k_{1}-1},} x_{0}\right)\right| \text {, }
\end{aligned}
$$

where $\max$ is taken for $\beta_{0} \leq \alpha_{0}, \beta_{k_{j}} \leq \alpha_{k_{j}}, \beta_{k_{j}-1} \leq M(m), 1 \leq j \leq r$, and sup is taken for $x_{k_{j}-1}, 1 \leq j \leq r$. Moreover, for any sequence of integers $k_{r}<l_{1}-1<l_{1}$ $<l_{2}-1<\cdots<l_{q} \leq L$, and for arbitrary multi-indices $\alpha_{L}, \alpha_{l_{u}}, \alpha_{l_{u}-1}, 1 \leq u$ $\leq q$, if $\alpha_{0}, \alpha_{k_{j}} \leq m, 1 \leq j \leq r$, then

$$
\mid \partial_{L}^{\alpha_{L}} \partial_{0}^{\alpha_{0}} \prod_{u=1}^{q}\left(\partial_{l_{u}}^{\alpha_{l_{u}}} \partial_{l_{u}-1}^{\alpha_{l_{u}-1}}\right) \prod_{j=1}^{r} \partial_{k_{j}}^{\alpha_{k j}}
$$




$$
\begin{aligned}
& \times p_{k_{r} k_{r-1} \ldots k_{1}}\left(\bar{x}_{L}, x_{l_{q}},{\widetilde{x_{l_{q}-1}}}_{x_{l_{q-1}}}, \ldots, \bar{x}_{l_{1}-1}, x_{k_{r}}, x_{k_{r-1}}, \ldots, x_{k_{1}}, x_{0}\right) \\
& \leq C_{m, 2}^{r} \prod_{j=1}^{r}\left(\frac{t_{k_{j}} T\left(k_{j}-1, k_{j-1}+1\right)}{\nu T\left(k_{j}, k_{j-1}+1\right)}\right) \\
& \times \max \sup \mid \partial_{L}^{\alpha_{L}} \partial_{0}^{\beta_{0}} \prod_{u=1}^{q}\left(\partial_{l_{u}-1}^{\alpha_{l_{u-1}}} \partial_{l_{u}}^{\alpha_{l_{u}}} \prod_{j=1}^{r} \partial_{k_{j}-1}^{\beta_{k_{j-1}-1}} \partial_{k_{j}}^{\beta_{k_{j}}}\right)
\end{aligned}
$$

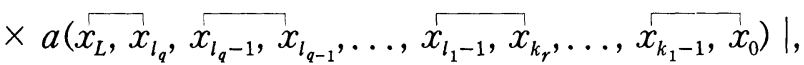

where max is taken for $\beta_{0} \leq \alpha_{0}, \beta_{k_{j}} \leq \alpha_{k,}, \beta_{k_{j-1}} \leq M(m), 1 \leq j \leq r$, and sup is taken for $x_{k_{j-1}}, 1 \leq j \leq r$. Here when $k_{r}=L$ and $l_{q}=L$ respectively, the notation $\partial_{L}^{\alpha_{L}}$ appears only once and we understand $x_{L}, x_{k_{r}}=x_{k_{r}}$ and $x_{L}, x_{l_{q}}=x_{l_{q}}$ on both the sides of the inequalities (5.18) and (5.19) respectively. We can choose $M(m)=2 m+4+2$.

Proof. We prove only (5.19) by induction on $r$. (5.18) will be shown similarly. To prove the case for $r=1$, we abbreviate $k_{1}$ as $k$. We have

$$
\begin{aligned}
p_{k}\left(x_{L}, x_{L-1}, \ldots, x_{k+1}, x_{k}, x_{0}\right) & =\left(R_{k-1} S_{k-2} \cdots S_{1} a\right)\left(x_{L}, x_{L-1}, \ldots, x_{k}, x_{0}\right), \quad k \geq 3, \\
& =\left(R_{1} a\right)\left(x_{L}, \ldots, x_{2}, x_{0}\right), \quad k=2 .
\end{aligned}
$$

We set

$$
\begin{aligned}
q\left(x_{L}, \ldots, x_{k}, x_{k-1}, x_{0}\right) & =\left(S_{k-2} \cdots S_{1} a\right)\left(x_{L}, x_{L-1}, \ldots, x_{k}, x_{k-1}, x_{0}\right) \\
& =D\left(x_{k-1}, x_{0}\right)^{-1 / 2} a\left(x_{L}, x_{L-1}, \ldots, x_{k}, x_{k-1}, x_{0}\right), \text { if } k \geq 3, \\
& =a\left(x_{L}, \ldots, x_{2}, x_{1}, x_{0}\right), \text { if } k=2 .
\end{aligned}
$$

Let $S_{1,1}^{\#}\left(x_{1}, x_{0}\right)=S_{1}\left(x_{1}, x_{0}\right)$. Then we have

$$
\begin{aligned}
& \left(\frac{E}{t_{k}}\right)^{1 / 2}\left(\frac{E}{T(k-1,1)}\right)^{1 / 2} \int_{\mathbf{R}} e^{-i \nu\left(S_{k}\left(x_{k}, x_{k-1}\right)+S_{k-1,1}^{\#}\left(x_{k-1}, x_{0}\right)\right)} \\
& \times q\left(x_{L}, x_{L-1}, \ldots, x_{k}, x_{k-1}, x_{0}\right) d x_{k-1} \\
& =\left(\frac{E}{T(k, 1)}\right)^{1 / 2} e^{-i \nu S_{k, 1}^{\#}\left(x_{k}, x_{0}\right)} \\
& \quad \times\left(D\left(S_{k}+S_{k-1,1}^{\#} ; x_{k}, x_{0}\right)^{-1 / 2} q\left(x_{L}, x_{L-1}, \ldots, x_{k}, x_{0}\right)+p_{k}\left(x_{L}, \ldots, x_{k}, x_{0}\right)\right) .
\end{aligned}
$$

Therefore, if $k<l_{1}-1<l_{1}<l_{2}-1<\cdots<l_{q} \leq L$, then

$$
\begin{aligned}
& \left(\frac{E}{t_{k}}\right)^{1 / 2}\left(\frac{E}{T(k-1,1)}\right)^{1 / 2} \int_{\mathbf{R}} e^{-i \nu\left(S_{k}\left(x_{k}, x_{k-1}\right)+S_{k-1,1}^{\#}\left(x_{k-1}, x_{0}\right)\right)} \\
& \times q\left(x_{L}, x_{l_{q}}, \widetilde{x_{l_{q}-1}, x_{l_{q-1}}}, \ldots, \widetilde{x_{l_{1}-1}, x_{k}}, x_{k-1}, x_{0}\right) d x_{k-1}
\end{aligned}
$$




$$
\begin{aligned}
& =\left(\frac{E}{T(k, 1)}\right)^{1 / 2} e^{-i \nu S_{k, 1}^{\#}\left(x_{k}, x_{0}\right)} \\
& \times\left(D\left(S_{k}+S_{k-1,1}^{\#} ; x_{k}, x_{0}\right)^{-1 / 2} q\left(\widetilde{x_{L}, x_{l_{q}}}, \widetilde{x_{l_{q}-1}, x_{l_{q-1}}}, \ldots, \widetilde{x_{l_{1}-1}, x_{k}}, x_{0}\right)\right.
\end{aligned}
$$

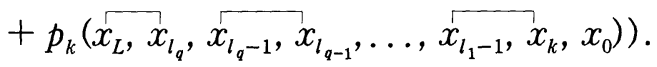

Differentiating (5.22) with respect to $x_{L}, x_{l_{u}}, x_{l_{u}-1}$ and applying the stationary phase method Lemma 4.1, we have the estimate: For any $m \geq 0$ there exists $C_{m}$ such that for arbitrary $\alpha_{l_{u}}, \alpha_{l_{u}-1}, \alpha_{L}$, if $\alpha_{0}, \alpha_{k} \leq m$,

$$
\begin{aligned}
& \left|\partial_{k}^{\alpha_{k}} \partial_{0}^{\alpha_{0}} \prod_{u=1}^{q}\left(\partial_{l_{u}}^{\alpha_{l_{u}}} \partial_{l_{u}-1}^{\alpha_{l_{u}-1}}\right) \partial_{L}^{\alpha_{L}} p_{k}\left(\widetilde{x_{L}, x_{l_{q}}}, \widetilde{x_{l_{q}-1}}, \ldots, \widetilde{x_{l_{1}-1}}, x_{k}, x_{0}\right)\right| \\
& \leq C_{m}\left(\frac{t_{k} T(k-1,1)}{\nu T(k, 1)}\right) \max \sup _{x_{k-1}} \mid \partial_{k}^{\beta_{k}} \partial_{k-1}^{\beta_{k-1}} \partial_{0}^{\beta_{0}} \prod_{u=1}^{q}\left(\partial_{l_{u}}^{\alpha_{l u}} \partial_{l_{u}-1}^{\alpha_{u^{-1}-1}}\right) \partial_{L}^{\alpha_{L}} \\
& \times q\left(\widetilde{x_{L}, x_{l_{q}}, \ldots, x_{l_{1}-1}, x_{k}}, x_{k-1}, x_{0}\right) \mid,
\end{aligned}
$$

where $\max$ is taken for $\beta_{0} \leq \alpha_{0}, \beta_{k} \leq \alpha_{k}, \beta_{k-1} \leq K(m)=2 m+4+2$. When $l_{q}=L$, the notation $\partial_{L}^{\alpha_{L}}$ appears only once on both the sides of this inequality. From (5.20) Leibnitz' rule gives

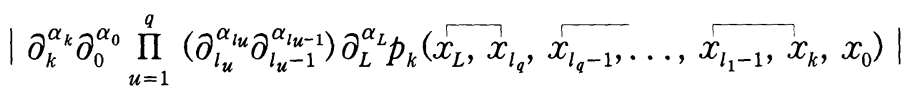

$$
\begin{aligned}
& \leq C_{m} C_{m}^{\prime}\left(\frac{t_{k} T(k-1,1)}{\nu T(k, 1)}\right) \max \sup _{x_{k-1}} \mid \partial_{k}^{\beta_{k}} \partial_{k-1}^{\beta_{k-1}} \partial_{0}^{\beta_{0}} \prod_{u=1}^{q}\left(\partial_{l_{u}}^{\alpha_{l_{u}}} \partial_{l_{u}-1}^{\alpha_{l_{u-1}}}\right) \partial_{L}^{\alpha_{L}}
\end{aligned}
$$

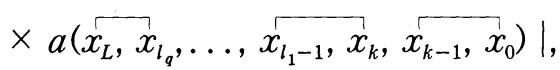

where $\max$ is taken for $\beta_{0} \leq \alpha_{0}, \beta_{k} \leq \alpha_{k}, \beta_{k-1} \leq K(m)=2 m+4+2$. We choose $C_{m, 2} \geq C_{m} C_{m}^{\prime}$. This proves (5.19) for $r=1$.

Next we suppose (5.19) for $r$ and prove it for $r+1$. Let $k_{r}<k_{r+1}-1$ $<k_{r+1}<l_{1}-1<l_{1}<\cdots<l_{q} \leq L$. We set

$$
\begin{aligned}
& q\left(x_{L}, \ldots, x_{k_{r+1}}, x_{k_{r+1}-1}, x_{k_{r}}, \ldots, x_{k_{1}}, x_{0}\right) \\
& =\left(S_{k_{r+1}-2} \cdots S_{k_{r}+1} p_{k_{r} \cdots k_{1}}\right)\left(x_{L}, \ldots, x_{k_{r+1}}, x_{k_{r+1}-1}, x_{k_{r}}, \ldots, x_{k_{1}}, x_{0}\right) . \\
& =D\left(x_{k_{r+1}-1}, x_{k_{r}}\right)^{-1 / 2} p_{k_{r} \cdots k_{1}}\left(x_{L}, \ldots, x_{k_{r+1}}, x_{k_{r+1}-1}, x_{k_{r}}, \ldots, x_{k_{1}}, x_{0}\right) .
\end{aligned}
$$

Then we have from (5.23)

$$
\begin{aligned}
& \left(\frac{E}{t_{k_{r+1}}}\right)^{1 / 2}\left(\frac{E}{T\left(k_{r+1}-1, k_{r}+1\right)}\right)^{1 / 2} \\
& \times \int_{\mathbf{R}} e^{-i \nu\left(S_{k_{r+1}}\left(x_{k_{r+1}}, x_{k_{r+1}-1}\right)+S_{k_{r+1}-1, k_{r}+1}^{\#}\left(x_{k_{r+1}-1}, x_{k \gamma}\right)\right)}
\end{aligned}
$$




$$
\begin{aligned}
& \times q\left(x_{L}, x_{l_{q}}, \ldots, x_{l_{1}-1}, x_{k_{r+1}}, x_{k_{r+1}-1}, x_{k_{r}}, \ldots, x_{k_{1}}, x_{0}\right) d x_{k_{r+1}-1} \\
& =\left(\frac{E}{T\left(k_{r+1}, k_{r}+1\right)}\right)^{1 / 2} e^{-\imath \nu S_{k_{r+1} k_{r}+1}^{*}\left(x_{k_{r+1}} x_{k_{r}}\right)} \\
& \times\left[D\left(S_{k_{r+1}}+S_{k_{r+1}-1, k_{r}+1}^{\#} ; x_{k_{r+1}}, x_{k_{\gamma}}\right)^{-1 / 2} q\left(x_{L}, x_{l_{q}}, \ldots, x_{l_{1}-1}, x_{k_{r+1}}, x_{k_{r}}, \ldots, x_{0}\right)\right.
\end{aligned}
$$

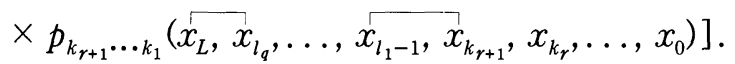

We apply Lemma 4.1 to (5.24). Then we have from (5.23) for any $m \geq 0$ if $\alpha_{k_{r}}$, $\alpha_{k_{r+1}} \leq m$,

$$
\begin{aligned}
& \left|\partial_{L}^{\alpha_{L}} \partial_{0}^{\alpha_{0}} \prod_{u=1}^{q}\left(\partial_{l_{u}}^{\alpha_{l u}} \partial_{l_{u}-1}^{\alpha_{l_{u}-1}}\right) \prod_{u=1}^{r+1}\left(\partial_{k_{u}}^{\alpha_{k u}}\right) p_{k_{r+1} \ldots k_{1}}\left(x_{L}, x_{l_{q}}, \ldots, x_{l_{1}-1}, x_{k_{r+1}}, x_{k_{r}}, \ldots, x_{0}\right)\right| \\
& \leq C_{m}\left(\frac{t_{k_{r+1}} T\left(k_{r+1}-1, k_{r}+1\right)}{\nu T\left(k_{r+1}, k_{r}+1\right)}\right) \\
& \times \max \sup _{x_{k+1-1}} \mid \partial_{L}^{\alpha_{L}} \partial_{0}^{\alpha_{0}} \prod_{u=1}^{q}\left(\partial_{l_{u}}^{\alpha_{l_{u}}} \partial_{l_{u}-1}^{\alpha_{l_{u}-1}}\right) \prod_{u=1}^{r-1}\left(\partial_{k_{u}}^{\alpha_{k u}}\right) \partial_{k_{r+1}}^{\beta_{k_{r+1}}} \partial_{k_{r+1}-1}^{\beta_{k_{r+1}-1}} \partial_{k_{r}}^{\beta_{k_{r}}} \\
& \times q\left(x_{L}, x_{l_{q}}, \ldots, x_{l_{1}-1}, x_{k_{r+1}}, x_{k_{r+1}-1}, x_{k_{r}}, \ldots, x_{k_{1}}, x_{0}\right) \mid, \\
& \leq C_{m} C_{m}^{\prime}\left(\frac{t_{k_{r+1}} T\left(k_{r+1}-1, k_{r}+1\right)}{\nu T\left(k_{r+1}, k_{r}+1\right)}\right) \\
& \times \max \sup _{x_{k_{r+1}-1}} \mid \partial_{L}^{\alpha_{L}} \partial_{0}^{\alpha_{0}} \prod_{u=1}^{q}\left(\partial_{l_{u}}^{\alpha_{l u}} \partial_{l_{u}-1}^{\alpha_{l u-1}}\right) \prod_{u=1}^{r-1}\left(\partial_{k_{u}}^{\alpha_{k u}}\right) \partial_{k_{r+1}}^{\beta_{k r+1}} \partial_{k_{r+1}-1}^{\beta_{k r+1-1}} \partial_{k_{r}}^{\beta_{k r}}
\end{aligned}
$$

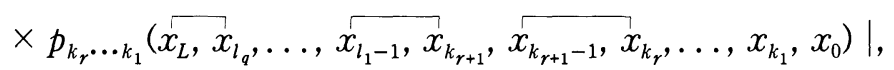

where max is taken for $\beta_{k_{r}} \leq \alpha_{k_{r}}, \beta_{k_{r+1}} \leq \alpha_{k_{r+1}}, \beta_{k_{r+1}-1} \leq K(m)=2 m+4+2$. If $l_{q}=L$, then $\partial_{L}^{\alpha_{L}}$ appears only once in any of the three members of (5.25). When we assume that $\alpha_{0}, \alpha_{k_{u}} \leq m, 1 \leq u \leq r+1$ as in Lemma 5.2, we can estimate for any $\alpha_{L}, \alpha_{l_{u}}, \alpha_{l_{u}-1}, 1 \leq u \leq r+1$ the last member of (5.25) by the induction hypothesis for $r$ where $q$ is replaced by $q+1$ and $\left(l_{1}, \ldots, l_{q}\right)$ is replaced by $\left(k_{r+1}, l_{1}, \ldots, l_{q}\right)$. Hence we have

$$
\begin{aligned}
& \left|\partial_{L}^{\alpha_{L}} \partial_{0}^{\alpha_{0}} \prod_{u=1}^{q}\left(\partial_{l_{u}}^{\alpha_{l_{u}}} \partial_{l_{u}-1}^{\alpha_{l_{u}-1}}\right) \prod_{u=1}^{r+1}\left(\partial_{k_{u}}^{\alpha_{k u}}\right) p_{k_{r+1} \ldots k_{1}}\left(\bar{x}_{L}, x_{l_{q}}, \ldots, \widetilde{x_{l_{1}-1}}, x_{k_{r+1}}, x_{k_{r}}, \ldots, x_{0}\right)\right| \\
& \leq C_{m} C_{m}^{\prime} C_{m, 2}^{r} \prod_{u=1}^{r+1}\left(\frac{t_{k_{u}} T\left(k_{u}-1, k_{u-1}+1\right)}{\nu T\left(k_{u}, k_{u-1}+1\right)}\right) \\
& \times \max \sup \mid \partial_{L}^{\alpha_{L}} \partial_{0}^{\alpha_{0}} \prod_{u=1}^{q}\left(\partial_{l_{u}}^{\alpha_{l_{u}}} \partial_{l_{u}-1}^{\alpha_{l_{u-1}}}\right) \prod_{u=1}^{r+1}\left(\partial_{k_{u}}^{\beta_{k_{u}}} \partial_{k_{u}-1}^{\beta_{k_{u}-1}}\right)
\end{aligned}
$$

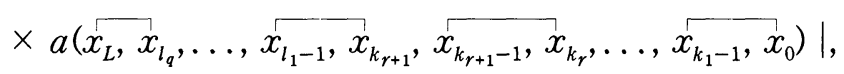


where max is taken for $\beta_{0} \leq \alpha_{0}, \beta_{k_{u}} \leq \alpha_{k_{u}}, \beta_{k_{u^{-1}}} \leq K(m)=2 m+4+2,1 \leq u$ $\leq r+1$ and sup is taken for $x_{k_{u}-1}$. Thus Lemma 5.2 has been proved.

Proof of Theorem 2. Let $a \equiv 1$ and $p_{j_{s} j_{s-1} \cdots j_{1}}$ be a function defined by (5.17) with $\left(j_{s}, \ldots, j_{1}\right)$ in place of $\left(k_{r}, \ldots, k_{1}\right)$.

Lemma 5.3. Let $T_{L}<\delta^{\prime}$. Then $p_{j_{s} j_{s-1} \ldots j_{1}}\left(x_{L}, x_{L-1}, \ldots, x_{j_{s}+1}, x_{j_{s}}, x_{j_{s-1}}, \ldots, x_{j_{1}}, x_{0}\right)$ is a function of only $\left(x_{j_{s}}, x_{j_{s-1}}, \ldots, x_{j_{1}}, x_{0}\right)$, i.e., $p_{j_{s} j_{s-1} \ldots j_{1}}$ is independent of $x_{k}, k \geq j_{s}$ +1 . It is of the form

$$
\begin{aligned}
& p_{j_{s} j_{s-1} \ldots j_{1}}\left(x_{L}, x_{L-1}, \ldots, x_{j_{s}+1}, x_{j_{s}}, x_{j_{s-1}}, \ldots, x_{j_{1}}, x_{0}\right) \\
& =\prod_{r=1}^{s} \nu^{-1} t_{j_{r}} T\left(j_{r}-1, j_{r-1}+1\right) p_{j_{r}}^{\prime}\left(x_{j_{r}}, x_{j_{r-1}}\right)
\end{aligned}
$$

where for any $\alpha, \beta$,

$$
\left|\partial_{j_{r}}^{\alpha} \partial_{j_{r-1}}^{\beta} p_{j_{r}}^{\prime}\left(x_{j_{r}}, x_{j_{r-1}}\right)\right| \leq C_{\alpha \beta} .
$$

Here the constants $C_{\alpha \beta}$ depend only on $\alpha, \beta$.

We note here that Lemma 5.3 differs from Fujiwara [5, Lemma 5.1] in the power of $T\left(j_{r}-1, j_{r-1}+1\right)$; our power is 1 while his is 2 . However, we shall be able to prove Lemma 5.3 in the same way as there. We only indicate here one different point. Namely, we have by Lemma 3.10

$$
D\left(x_{j-1}, x_{0}\right)^{-1 / 2}=1+T(j-1,1) q_{j-1}\left(x_{j-1}, x_{0}\right),
$$

for some $q_{j-1}\left(x_{j-1}, x_{0}\right) \in \mathscr{B}(\mathbf{R} \times \mathbf{R})$, where the power of $T(j-1,1)$ is 1 , not 2 .

The proof of Theorem 2 will also proceed in the same way as in $[5, \S 5]$. We have

$$
\begin{aligned}
b_{j_{s} j_{s-1} \cdots j_{1}} & =S_{L-1} S_{L-1} \cdots S_{j_{s+1}} p_{j_{s} j_{s-1} \cdots j_{1}} \\
& =D\left(x_{L}, x_{j_{s}}\right)^{-1 / 2} p_{j_{s} j_{s-1} \cdots j_{1}},
\end{aligned}
$$

where if $j_{s}=L$ or $L-1$, then $D\left(x_{L}, x_{j_{s}}\right)=1$. So we combine Lemma 5.3 with (5.14a, b) to obtain that if $\alpha_{0}, \alpha_{L} \leq m$,

$$
\begin{aligned}
& \quad\left|\partial_{L}^{\alpha_{L}} \partial_{0}^{\alpha_{0}} a_{j_{s} j_{s-1} \cdots j_{1}}\left(x_{L}, x_{0}\right)\right| \\
& \leq C_{m}^{s} \max \sup _{x_{j_{u}}, u=1, \cdots, s} \mid \partial_{L}^{\beta_{L}} \prod_{r=1}^{s} \partial_{j_{r}}^{\beta_{j_{r}}} \partial_{0}^{\beta_{0}} \\
& \quad \times D\left(x_{L}, x_{j_{s}}\right)^{-1 / 2} \prod_{r=1}^{s} \nu^{-1} t_{j_{r}} T\left(j_{r}-1, j_{r-1}+1\right) p_{j_{r}}^{\prime}\left(x_{j_{r}}, x_{j_{r-1}}\right) \mid
\end{aligned}
$$




$$
\leq C_{m, 1}^{s} \prod_{r=1}^{s}\left(\nu^{-1} t_{j_{r}} T\left(j_{r}-1, j_{r-1}+1\right)\right) .
$$

Therefore, from (5.15) we have

$$
\begin{aligned}
\left|\partial_{L}^{\alpha_{L}} \partial_{0}^{\alpha_{0}} r\left(x_{L}, x_{0}\right)\right| & \leq \sum^{\prime} \prod_{r=1}^{s}\left(C_{m, 2} \nu^{-1} T_{L}\right) t_{\jmath_{r}} \\
& \leq \prod_{j=1}^{L}\left(1+C_{m, 2} \nu^{-1} T_{L} t_{j}\right)-1 .
\end{aligned}
$$

This is the estimate (1.11) of Theorem 2.

Acknowledgement. The author would like to express his hearty thanks to Professor T. Ichinose for his constant encouragements and stimulating comments. He is also grateful to the referee for several useful comments.

\section{REFERENCES}

[1] K. Asada, D. Fujiwara, On some oscillatory integral transformations in $L^{2}\left(\mathbf{R}^{n}\right)$, Japan J. Math., 4 (1978), 299-361.

[2] R. P. Feynman, Space time approach to non-relativistic quantum mechanics, Rev. Modern Phys., 20 (1948), 367-386.

[ 3 ] D. Fujiwara, Remarks on convergence of some Feynman path integrals, Duke Math. J., 47 (1980), 559-600.

[4] D. Fujiwara, A remark on Taniguchi-Kumanogo theorem for product of Fourier integral operators, Pseudo-differential operators, Proc. Oberwolfach 1986, Lecture Notes in Math., 1256, Springer, 135-153 (1987).

[5] D. Fujiwara, The stationary phase method with an estimate of the remainder term on a space of large dimension, Nagoya Math. J., 124 (1991), 61-97.

[6] D. Fujiwara, Some Feynman path integrals as oscillatory integrals over a Sobolev manifold, Lecture Notes in Math., 1540, Springer (1993), 39-53.

[ 7 ] H. Kumanogo, Pseudo-differential Operators, MIT Press, 1982.

[8] F. Nicoleau, Approximation semi-classique du propagateur d'un système èlectromagnétique et phénomène de Aharonov-Bohm, Helv. Phys. Acta., 65 (1992), $722-747$.

[9] K. Yajima, Schrödinger evolution equations with magnetic fields, J. Analyse Math., $\mathbf{5 6}$ (1991), 29-76.

Department of Mathematics

Faculty of Science

Kanazawa University

920-11 Kanazawa, Japan 